\title{
Stieltjes differential systems with nonmonotonic derivators
}

\author{
Marlène Frigon ${ }^{1}$ and F. Adrián F. Tojo ${ }^{2^{*}}$ (1)
}

\author{
"Correspondence: \\ fernandoadrian.fernandez@usc.es \\ ${ }^{2}$ Instituto de Matemáticas, \\ Facultade de Matemáticas, \\ Universidade de Santiago de \\ Compostela, Santiago de \\ Compostela, Spain \\ Full list of author information is \\ available at the end of the article
}

\begin{abstract}
In this work we study Stieltjes differential systems of which the derivators are allowed to change sign. This leads to the definition of the notion of a function of controlled variation, a characterization of precompact sets of $g$-continuous functions, and an explicit expression of $g$-exponential maps. Finally, we prove a Peano-type existence result and apply it to a model of fluid stratification on buoyant miscible jets and plumes.
\end{abstract}

MSC: 34A37; 34L30; 26A42; 46B50; 37N 10

Keywords: Stieltjes derivatives; Differential systems; Fundamental Theorem of Calculus; Ascoli-Arzelà Theorem; Fluid stratification

\section{Introduction}

In the last years, there has been quite an interest in the theory regarding problems with Stieltjes derivatives. This kind of problems can be traced back to the works of Kurzweil [11] and later of Schwabik and Tvrdý $[17,20,21]$ but, whereas these works deal with Stieltjes measures directly through the associated integral problems, some others, such as $[7,12$, 14], center their attention in the definition and the meaning of the Stieltjes derivatives.

In a recent article of López Pouso and Márquez Albés [13], the authors study the case of systems with Stieltjes derivatives with several different derivators. All of them are assumed to be left-continuous and nondecreasing. They establish the existence of a solution of the following system of differential equations with respect to $h$ :

$$
x_{h}^{\prime}(t)=f(t, x(t)) \quad \mu_{h} \text {-a.e. } t \in\left[t_{0}, t_{0}+\tau\right], x(0)=x_{0},
$$

where $h=\left(h_{1}, \ldots, h_{n}\right):\left[t_{0}, t_{0}+T\right] \rightarrow \mathbb{R}^{n}$ is such that $h_{i}$ is left-continuous and nondecreasing for every $i=1, \ldots, n$ (see [13, Theorem 4.5]).

In this manuscript, we consider more general maps $h$. More precisely, we want to see if the nondecreasingness assumption on all $h_{i}$ can be dropped. In particular, for $g:\left[t_{0}, t_{0}+\right.$ $\tau] \rightarrow \mathbb{R}$ a left-continuous function of bounded variation, we study the problem

$$
x_{g}^{\prime}(t)=f(t, x(t)) \quad \mu_{g} \text {-a.e. } t \in\left[t_{0}, t_{0}+\tau\right], x(0)=x_{0} .
$$

(c) The Author(s) 2020. This article is licensed under a Creative Commons Attribution 4.0 International License, which permits use, sharing, adaptation, distribution and reproduction in any medium or format, as long as you give appropriate credit to the original author(s) and the source, provide a link to the Creative Commons licence, and indicate if changes were made. The images or other third party material in this article are included in the article's Creative Commons licence, unless indicated otherwise in a credit line to the material. If material is not included in the article's Creative Commons licence and your intended use is not permitted by statutory regulation or exceeds the permitted use, you will need to obtain permission directly from the copyright holder. To view a copy of this licence, visit http://creativecommons.org/licenses/by/4.0/. 
First, let us observe that it is much easier to establish the existence of a solution of the weaker integral equation:

$$
x(t)=x_{0}+\int_{a}^{t} f(s, x(s)) \mathrm{d} \mu_{g}(s)
$$

where $\mu_{g}$ is the Lebesgue-Stieltjes measure with respect to $g$. Indeed, it is well known that there exist two left-continuous nondecreasing functions $g_{+}, g_{-}$such that $g=g_{+}-g_{-}$. This remark leads us to consider the following system:

$$
\begin{aligned}
& y_{g_{+}}^{\prime}(t)=f(t, y(t)+z(t)), \\
& z_{g_{-}}^{\prime}(t)=-f(t, y(t)+z(t)), \\
& \left(y\left(t_{0}\right), z\left(t_{0}\right)\right)=\left(x_{0}, 0\right) .
\end{aligned}
$$

For $h=\left(g_{+}, g_{-}\right)$, [13, Theorem 4.5], ensures the existence of a solution $(y, z)$ of (1.4). It is easy to see that $(y, z)$ is also a solution of the integral system:

$$
\begin{aligned}
& y(t)=x_{0}+\int_{a}^{t} f(t, y(t)+z(t)) \mathrm{d} \mu_{g_{+}}, \\
& z(t)=-\int_{a}^{t} f(t, y(t)+z(t)) \mathrm{d} \mu_{g_{-}} .
\end{aligned}
$$

So, $y+z$ satisfies

$$
(y+z)(t)=x_{0}+\int_{a}^{t} f(t, y(t)+z(t)) \mathrm{d}\left(\mu_{g_{+}}-\mu_{g_{-}}\right),
$$

that is, $x=y+z$ is a solution of (1.3).

It is important to realize that, although the previous procedure is simple, it is lacking in that it does not provide any information on the form or behavior of the $g$-derivative, and that is because we only obtained a solution of the integral equation (1.3). What is more, in order to study the problem (1.2), we would first have to define the $g$-derivative, the signed measure $\mu_{g}$, and also the positive measure $\left|\mu_{g}\right|$ in this context. Of course, the RadonNikodym derivative will be properly defined as it follows from the integral equation, but it lacks an interpretation in terms of $g$. In this sense, there have been previous treatments of derivatives with respect to functions of bounded variation from the measure perspective (see [4, 8]) or related to the Kurzweil-Stieltjes integral [16]. Measures have the great advantage of allowing for a general treatment without worrying about the behavior at specific points, but this same asset turns into a disadvantage when it is precisely that trait that we want to control in practical applications.

It is for the reasons mentioned above that we will study the case of systems with $g$ derivators that can change sign, focusing on the obtaining of solutions of a system of $g$ differential equations. In order to achieve this goal, we will have to rework the theory regarding the Fundamental Theorem of Calculus for the Lebesgue-Stieltjes integral as presented in [14], where it was done only for measures associated to nondecreasing functions. Allowing our derivators to be nonmonotonic will force us to introduce new concepts, such as that of a function of controlled variation, in order to correctly define a new notion of 
absolute continuity which is not based on related measures (see [8]) but relies directly on the function.

This development of the theory will allow us to obtain an explicit formula for the $g$ exponential map which will be the solution of a linear $g$-differential equation. Then, we will present an analog of the Peano theorem in this context. Finally, in the last section, we show how the theory of $g$-differential equations with nonmonotone $g$ can be applied to a model of fluid stratification on buoyant miscible jets and plumes presented in [2].

\section{Preliminaries}

We recall some facts about bounded variation functions and their relation with signed measures.

Definition 2.1 Let $I \subset \mathbb{R}$ be an interval and $g: I \rightarrow \mathbb{R}$. Given an interval $J \subset I$, we denote

$$
\mathcal{P}(J):=\left\{P=\left(x_{1}, \ldots, x_{k_{P}}\right): k_{P} \geq 2 ; x_{j} \in J, j=1, \ldots, k_{P} ; x_{j} \leq x_{j+1}, j=1, \ldots, k_{P}-1\right\} .
$$

The total variation of $g$ in $J$ is defined by

$$
\operatorname{var}_{g} J:=\sup _{P \in \mathcal{P}())} \sum_{j=1}^{k_{P}-1}\left|g\left(x_{j+1}\right)-g\left(x_{j}\right)\right| \in[0, \infty] .
$$

If $\operatorname{var}_{g}(I)<\infty$, we say that $g$ is a function of bounded variation and we denote

$$
\begin{aligned}
& \mathrm{BV}(I, \mathbb{R})=\{g: I \rightarrow \mathbb{R}: g \text { is a function of bounded variation }\}, \\
& \mathrm{BV}_{-}(I, \mathbb{R})=\{g \in \mathrm{BV}(I, \mathbb{R}): g \text { is left continuous }\} .
\end{aligned}
$$

From now on we write $g\left(x^{+}\right):=\lim _{y \rightarrow x^{+}} g(y)$ and $g\left(x^{-}\right):=\lim _{y \rightarrow x^{-}} g(y)$. In order to avoid having to separate cases when $x$ is an extremity of $I$, for instance, in the case $I=[a, b]$, we define $g\left(a^{-}\right):=g(a)$ and $g\left(b^{+}\right):=g(b)$. It is well known that functions of bounded variation are regulated, so lateral limits always exist. For $g \in \mathrm{BV}(I, \mathbb{R})$, let us define

$$
\begin{aligned}
& D_{g}^{+}:=\left\{x \in I: g\left(x^{+}\right)-g\left(x^{-}\right)>0\right\}, \\
& D_{g}^{-}:=\left\{x \in I: g\left(x^{+}\right)-g\left(x^{-}\right)<0\right\}, \\
& D_{g}:=D_{g}^{+} \cup D_{g}^{-},
\end{aligned}
$$

and

$$
C_{g}:=\left\{x \in I: g(x)=g(y), y \in(x-\varepsilon, x+\varepsilon) \text { for some } \varepsilon \in \mathbb{R}^{+}\right\} .
$$

It is also well known that $D_{g}$ is countable [14]. Given an interval $J \subset I$, we consider

$$
\begin{aligned}
& \operatorname{var}_{g}^{+} J=\sup _{P \in \mathcal{P}(J)} \sum_{j=1}^{k_{P}-1}\left[g\left(x_{j+1}\right)-g\left(x_{j}\right)\right]^{+}, \\
& \operatorname{var}_{g}^{-} J=\sup _{P \in \mathcal{P}(J)} \sum_{j=1}^{k_{P}-1}\left[g\left(x_{j+1}\right)-g\left(x_{j}\right)\right]^{-},
\end{aligned}
$$


where $c^{+}=\max \{c, 0\}$ and $c^{-}=-\min \{c, 0\}$. We call $\operatorname{var}_{g}^{+} J$ and $\operatorname{var}_{g}^{-} J$ the positive variation and the negative variation of $g$ on $J$, respectively.

For $g \in \mathrm{BV}_{-}(I, \mathbb{R})$, $\operatorname{var}_{g}^{+}$, $\operatorname{var}_{g}^{-}$, and $\operatorname{var}_{g}$ can serve to construct measures $\mu_{g}^{+}, \mu_{g}^{-}$, and $\left|\mu_{g}\right|$, respectively, [1, Theorem 4.1.9] in the following way. Define, for instance, $v_{g}(x):=$ $\operatorname{var}_{g}[a, x), x \in(a, b]$. Thus defined, $v_{g}$ is left continuous and there is a unique measure, $\left|\mu_{g}\right|$, such that

$$
\left|\mu_{g}\right|([x, y))=v_{g}(y)-v_{g}(x)=\operatorname{var}_{g}[a, y)-\operatorname{var}_{g}[a, x)=\operatorname{var}_{g}[x, y) .
$$

Observe that

$$
\left|\mu_{g}\right|(\{x\})=v_{g}\left(x^{+}\right)-v_{g}(x)=: \operatorname{var}_{g}\left[x, x^{+}\right]=g\left(x^{+}\right)-g(x) .
$$

So, $\left|\mu_{g}\right|(\{x\}) \neq 0$ if and only if $x \in D_{g}$. Note that $C_{g}$ is, by definition, an open set in the usual topology of $I$. Therefore, it can be rewritten uniquely as the disjoint countable union of open intervals, say $C_{g}=\bigcup_{n \in \mathbb{N}}\left(a_{n}, b_{n}\right)$. Thus, it is easy to see that $\left|\mu_{g}\right|\left(C_{g}\right)=0$. The measures $\mu_{g}^{+}$and $\mu_{g}^{-}$are defined similarly from $\operatorname{var}_{g}^{-}$and $\operatorname{var}_{g}^{+}$, respectively.

Lemma 2.2 The measure $|\mu|_{g}$ satisfies $\left|\mu_{g}\right|=\mu_{g}^{+}+\mu_{g}^{-}$.

Definition 2.3 (Jordan Decomposition) We define the signed measure $\mu_{g}:=\mu_{g}^{+}-\mu_{g}^{-}$.

The definitions presented here for $\mu_{g}^{+}, \mu_{g}^{-}$, and $\left|\mu_{g}\right|$ coincide with those stated in order to obtain the Jordan decomposition [1, Definition 5.1.2], that is, for any $\mu_{g}$-measurable set $A$, we have that

$$
\begin{aligned}
& \mu_{g}^{+}(A)=\sup \left\{\mu_{g}(B): B \subset A, B \mu_{g} \text {-measurable }\right\} \\
& \mu_{g}^{-}(A)=\sup \left\{-\mu_{g}(B): B \subset A, B \mu_{g} \text {-measurable }\right\} \\
& \left|\mu_{g}\right|(A)=\mu_{g}^{+}(A)+\mu_{g}^{-}(A) .
\end{aligned}
$$

We denote by $\mathrm{L}_{g}^{1}(I, \mathbb{R})$ the space of $\left|\mu_{g}\right|$-integrable functions on the interval $I$, that is, the set of $\left|\mu_{g}\right|$-measurable maps $h: I \rightarrow \mathbb{R}$ such that $\int_{I}|h| \mathrm{d}\left|\mu_{g}\right|<\infty$. Notice that

$$
\int_{I} h \mathrm{~d}\left|\mu_{g}\right|=\int_{I \backslash D_{g}} h \mathrm{~d}\left|\mu_{g}\right|+\sum_{t \in D_{g}} h(t)\left|g\left(t^{+}\right)-g(t)\right| .
$$

Remark 2.4 Observe that if a function is $\left|\mu_{g}\right|$-integrable, it is also $\mu_{g}$-integrable.

\section{Functions of controlled variation}

In this section, we introduce the notion of functions of controlled variation.

Definition 3.1 We say that $g \in \mathrm{BV}_{-}(I, \mathbb{R})$ is a function of controlled variation if there exists a closed set $F_{g} \subset I$ such that $g$ is monotonic on each connected component of $I \backslash F_{g}$ and $\left|\mu_{g}\right|\left(F_{g} \backslash D_{g}\right)=0$. We denote

$$
\mathrm{CV}_{-}(I, \mathbb{R})=\left\{g \in \mathrm{BV}_{-}(I, \mathbb{R}): g \text { is a function of controlled variation }\right\} \text {. }
$$


Proposition 3.2 The set $\mathrm{CV}_{-}(I, \mathbb{R})$ is a dense subset of $\mathrm{BV}_{-}(I, \mathbb{R})$.

Proof Rational polynomials are contained in $\mathrm{CV}_{-}(I, \mathbb{R})$ and are dense in $\mathrm{L}^{1}(I, \mathbb{R})$. There is a continuous inclusion of $\mathrm{BV}(I, \mathbb{R})$ into $\mathrm{L}^{1}(I, \mathbb{R})[10]$, so $\mathrm{CV}_{-}(I, \mathbb{R})$ is dense in $\mathrm{BV}_{-}(I, \mathbb{R})$.

Remark 3.3 Not all functions in $\mathrm{BV}_{-}(I, \mathbb{R})$ are of controlled variation. In [5, Sect. 3], they build a continuous function $g$ of bounded variation that is not monotonic type on any interval. This implies that $F_{g}$ would have to be taken equal to $I$, but in that case $\left|\mu_{g}\right|\left(F_{g} \backslash D_{g}\right)=$ $\left|\mu_{g}\right|(I) \neq 0$, so $g \notin \mathrm{CV}_{-}(I, \mathbb{R})$. Given $g \in \mathrm{BV}_{-}(I, \mathbb{R})$, we have that $g=g_{1}-g_{2}$, where $g_{1}$ and $g_{2}$ are continuous nondecreasing functions, and therefore $g_{1}, g_{2} \in \mathrm{CV}_{-}(I, \mathbb{R})$. Hence, we deduce that $\mathrm{CV}_{-}(I, \mathbb{R})$ is not a vector space. Such functions in $\mathrm{BV}_{-}(I, \mathbb{R}) \backslash \mathrm{CV}_{-}(I, \mathbb{R})$ do not occur in practical applications and are only of interest as counterexamples.

In Remark 5.3, we will stress why it is important for $g$ to be of controlled variation.

Remark 3.4 Observe that, for $g \in \mathrm{CV}_{-}(I, \mathbb{R})$, the role of $F_{g}$ in the previous definition could be played by $F_{g} \backslash C_{g}$, which is compact and such that $\left(F_{g} \backslash C_{g}\right) \backslash D_{g}$ is hereditarily disconnected (that is, all of its connected components consist of just one point [9, p. 223]). To see this, take a connected component $E$ of $F_{g} \backslash D_{g}$ with more than a point. The connected components in the usual topology of $I$ are intervals and, since $\left|\mu_{g}\right|(E)=0, g$ has to be constant on $E$. Therefore, $\stackrel{\circ}{E}$ can be subtracted from $F_{g}$ because $g$ is monotonic on $E$ and $F_{g} \backslash \stackrel{\circ}{E}$ is closed. The set $E \backslash \stackrel{\circ}{E}$ has at most two points, each one is a connected component of $\left(F_{g} \backslash D_{g}\right) \backslash \stackrel{\circ}{E}$. We can do this process with all connected components of $F_{g} \backslash D_{g}$ with more than one point. We arrive to a new $F_{g}$, which is precisely $F_{g} \backslash C_{g}$, and $\left(F_{g} \backslash C_{g}\right) \backslash D_{g}$ is hereditarily disconnected. Moreover, $\left|\mu_{g}\right|\left(\left(F_{g} \backslash C_{g}\right) \backslash D_{g}\right)=\left|\mu_{g}\right|\left(F_{g} \backslash\left(D_{g} \cup C_{g}\right)\right)=0$.

Remark 3.5 Hereditarily disconnected sets, such as the Cantor set, can be uncountable.

In what follows, for $g \in \mathrm{CV}_{-}(I, \mathbb{R})$, we will assume that its associated closed set $F_{g}$ is a subset of $I \backslash C_{g}$.

Lemma 3.6 If $g \in \mathrm{CV}_{-}(I, \mathbb{R})$, then there exists a countable family of pairwise disjoint subintervals of $I,\left\{I_{\lambda}\right\}_{\lambda \in \Lambda}$, such that $F_{g}=I \backslash \bigcup_{\lambda \in \Lambda} I_{\lambda}$ and, for each $\lambda \in \Lambda, g$ is monotonic on $I_{\lambda}=\left(a_{\lambda}, b_{\lambda}\right)$.

Proof We assume, using Remark 3.4, that $F_{g} \backslash D_{g}$ is hereditarily disconnected. Since $F_{g}$ is closed, the open set $\mathbb{R} \backslash F_{g}$ is a (unique) disjoint union of a countable (because the usual topology of $\mathbb{R}$ is second countable) family of pairwise disjoint open intervals, $\bigcup_{\lambda \in \Lambda} I_{\lambda}$, where $I_{\lambda}=\left(a_{\lambda}, b_{\lambda}\right)$ for $\lambda \in \Lambda$. Since each $\left(a_{\lambda}, b_{\lambda}\right)$ is connected and contained in $I \backslash F_{g}, g$ is monotonic on $\left(a_{\lambda}, b_{\lambda}\right)$.

Definition 3.7 Let $g \in \mathrm{CV}_{-}(I, \mathbb{R})$ and its associated closed set $F_{g}=I \backslash \bigcup_{\lambda \in \Lambda} I_{\lambda} \subset I \backslash C_{g}$ with $\left\{I_{\lambda}\right\}_{\lambda \in \Lambda}$ a countable family of pairwise disjoint open intervals such that, for each $\lambda \in \Lambda, g$ is monotonic on $I_{\lambda}$. We define

$$
\begin{aligned}
& \Lambda^{+}=\left\{\lambda \in \Lambda: g \text { is nondecreasing on } I_{\lambda}=\left(a_{\lambda}, b_{\lambda}\right)\right\}, \quad \Lambda^{-}=\Lambda \backslash \Lambda^{+}, \\
& A^{+}=\bigcup_{\lambda^{+} \in \Lambda^{+}} I_{\lambda^{+}}, \quad A^{-}=\bigcup_{\lambda^{-} \in \Lambda^{-}} I_{\lambda^{-}} .
\end{aligned}
$$


Observe that $\mu_{g}^{+}\left(A^{-}\right)=\mu_{g}^{+}\left(D_{g}^{-}\right)=0$ and $\mu_{g}^{-}\left(A^{+}\right)=\mu_{g}^{-}\left(D_{g}^{+}\right)=0$.

Let us recall the existence of a Hahn decomposition associated to $g$.

Theorem 3.8 (Hahn Decomposition, [1, Theorem 5.1.6]) There exists a $\mu_{g}$-measurable set, $P \subset I$, such that $\mu_{g}^{+}(E)=\mu_{g}(E \cap P)$ and $\mu_{g}^{-}(E)=-\mu_{g}(E \backslash P)$ for every $E \subset I \mu_{g}$ measurable.

The sets given in Definition 3.7 are related to the Hahn decomposition associated to $\mu_{g}$.

Proposition 3.9 Let $g \in \mathrm{CV}_{-}(I, \mathbb{R})$ and $P \subset I$ the $\mu_{g}$-measurable set given by the Hahn decomposition of $\mu_{g}$. We have, for every $E \subset I \mu_{g}$-measurable,

$$
\begin{aligned}
& \mu_{g}^{+}(E)=\mu_{g}(E \cap P)=\mu_{g}\left(E \cap\left(A_{g}^{+} \cup D_{g}^{+}\right)\right) \quad \text { and } \\
& \mu_{g}^{-}(E)=-\mu_{g}(E \backslash P)=-\mu_{g}\left(\left(E \cap\left(A_{g}^{-} \cup D_{g}^{-}\right)\right) .\right.
\end{aligned}
$$

Proof Let $E \subset I \mu_{g}$-measurable. Since $\left|\mu_{g}\right|\left(F_{g} \backslash D_{g}\right)=0$,

$$
\begin{aligned}
\mu_{g}(E \cap P) & =\mu_{g}^{+}(E) \\
& =\mu_{g}^{+}\left(E \cap\left(F_{g} \backslash D_{g}\right)\right)+\mu_{g}^{+}\left(E \cap\left(A_{g}^{+} \cup D_{g}^{+}\right)\right)+\mu_{g}^{+}\left(E \cap\left(A_{g}^{-} \cup D_{g}^{-}\right)\right) \\
& =\mu_{g}^{+}\left(E \cap\left(A_{g}^{+} \cup D_{g}^{+}\right)\right) .
\end{aligned}
$$

Similarly, we deduce that $\mu_{g}^{-}(E)=-\mu_{g}(E \backslash P)=-\mu_{g}\left(E \cap\left(A_{g}^{-} \cup D_{g}^{-}\right)\right)$.

\section{The $g$-continuity and the relative compactness in $\mathrm{BC}_{g}(I, \mathbb{R})$}

From now on and unless stated otherwise, we will consider $g \in \mathrm{CV}_{-}(I, \mathbb{R})$ and $F_{g} \subset I \backslash C_{g}$ its associated closed set.

As notation, consider $[x, y]:=[y, x]$ if $y \leq x$. If we define $\Delta_{g}(x, y)=\operatorname{var}_{g}[x, y]$ for $x, y \in I$, we find that $\Delta$ is a displacement.

Definition 4.1 ([15]) Let $X \neq \emptyset$ be a set. A displacement is a function $\Delta: X^{2} \rightarrow \mathbb{R}$ such that the following properties hold:

(H1) $\Delta(x, x)=0, x \in X$.

(H2) For all $x, y \in X$,

$$
\varliminf_{z-y}|\Delta(x, z)|=|\Delta(x, y)|,
$$

where

$$
\varliminf_{z \rightarrow y}|\Delta(x, z)|:=\sup \left\{\liminf _{n \rightarrow \infty}\left|\Delta\left(x, z_{n}\right)\right|:\left(z_{n}\right)_{n \in \mathbb{N}} \subset X,\left|\Delta\left(y, z_{n}\right)\right| \stackrel{n \rightarrow \infty}{\longrightarrow} 0\right\}
$$

and the limit is considered in the usual topology of $\mathbb{R}$.

The fact that $\Delta_{g}$ is a displacement implies that we already know a lot regarding the topology generated by $g$. 
Definition 4.2 Given $t \in I$ and $r \in \mathbb{R}^{+}$, we define the g-open ball of center $t$ and radius $r$, as $B_{g}(t, r):=\left\{s \in I: \operatorname{var}_{g}[t, s]<r\right\}$. Also, we define the $g$-topology in the following way:

$$
\tau_{g}:=\left\{U \subset I: \forall t \in U \exists r \in \mathbb{R}^{+}, B_{g}(t, r) \subset U\right\} .
$$

Remark 4.3 This definition coincides with [7, Definition 3.1] when $g$ is nondecreasing.

When necessary, we will denote by $\tau_{u}$ the usual topology on $\mathbb{R}^{n}$.

Definition 4.4 A map $h: I \rightarrow \mathbb{R}$ is said to be $g$-continuous if $h:\left(I, \tau_{g}\right) \rightarrow\left(\mathbb{R}, \tau_{u}\right)$ is continuous. We denote by $\mathcal{C}_{g}(I, \mathbb{R})$ the set of $g$-continuous functions and by $\mathrm{BC}_{g}(I, \mathbb{R})$ the set of bounded $g$-continuous functions.

The proof of the following result is analogous to [7, Theorem 3.4].

Proposition 4.5 The space $\mathrm{BC}_{g}(I, \mathbb{R})$ endowed with the supremum norm is a Banach space.

As usual, $g$-continuity can be characterized using $g$-open balls.

Lemma 4.6 ([15]) A map $h: I \rightarrow \mathbb{R}$ is $g$-continuous if and only if, for every $t \in I$ and $\varepsilon \in \mathbb{R}^{+}$, there exists $\delta \in \mathbb{R}^{+}$satisfying $|h(s)-h(t)|<\varepsilon$ for every $s \in B_{g}(t, \delta)$.

From now on $I$ will be a compact interval $[a, b]$ in $\mathbb{R}$.

The next proposition can be proven as in [7, Proposition 3.2].

Proposition 4.7 If $g \in \mathrm{CV}_{-}(I, \mathbb{R})$ and $h: I \rightarrow \mathbb{R}$ is $g$-continuous, then

(1) $h$ is continuous from the left at every $t \in(a, b]$;

(2) if $g$ is continuous at $t \in[a, b)$, then so is $h$;

(3) if $g$ is constant on some $[\alpha, \beta] \subset I$, then so is $h$.

In [7], the authors gave sufficient conditions on certain sets of regulated functions in $\mathrm{BC}_{g}(I, \mathbb{R})$ for the case where $g$ is nondecreasing to ensure that they are relatively compact. Here, we provide a characterization for $\mathrm{BC}_{g}(I, \mathbb{R})$ in our more general setting using Vala's Theorem [22].

Definition 4.8 Let $X$ be a set and $(Y, d)$ be a metric space. We say that a map $f: X \rightarrow Y$ is precompact if $f(X)$ is precompact. Let $K(X, Y)$ be the family of precompact maps from $X$ to $Y$ endowed with the usual metric induced by $d$.

Theorem 4.9 (Ascoli-Arzelà-Vala [22, Theorem 1]) Let $K(X, Y)$ be endowed with the topology induced by the metric and let $S \subset K(X, Y)$. Then $S$ is precompact if and only if

(i) $S(x):=\{f(x) \in Y: f \in S\}$ is precompact for all $x \in X$;

(ii) $S$ is of equal variation, i.e., for every $\varepsilon \in \mathbb{R}^{+}$, there is a finite covering $\left\{X_{k}\right\}_{k=1}^{m}$ of $X$ such that $d(f(x), f(y))<\varepsilon$ for every $f \in S$, every $x, y \in X_{k}$ and every $k=1, \ldots, m$.

Now, all we have to do is to translate this characterization to the space $\mathrm{BC}_{g}(I, \mathbb{R})$. To this end, first observe that, since $\mathbb{R}$ is a complete space, a subset $A \subset \mathbb{R}$ is precompact 
if and only if it is relatively compact [6, (3.17.5)]. Furthermore, $\mathbb{R}$ satisfies Heine-Borel's property, so $A$ is precompact if and only if it is bounded. This implies that

$$
\mathrm{BC}_{g}(I, \mathbb{R})=\mathrm{BC}\left(\left(I, \tau_{g}\right),\left(\mathbb{R}, \tau_{u}\right)\right) \subset K\left(\left(I, \tau_{g}\right),(\mathbb{R},|\cdot|)\right),
$$

and, therefore, Theorem 4.9 is applicable to $\mathrm{BC}_{g}(I, \mathbb{R})$.

Definition 4.10 A set $S \subset \mathrm{BC}_{g}(I, \mathbb{R})$ is said to be $g$-equicontinuous if, for every $\varepsilon \in \mathbb{R}^{+}$and $t \in I$, there exists $\delta \in \mathbb{R}$ such that $|f(t)-f(s)|<\varepsilon$ for every $f \in S$ and every $s \in I$ such that $\operatorname{var}_{g}[t, s]<\delta$. We say that $S$ is uniformly g-equicontinuous if, for every $\varepsilon \in \mathbb{R}^{+}$, there exists $\delta \in \mathbb{R}^{+}$such that $|f(t)-f(s)|<\varepsilon$ for every $f \in S$ and every $t, s \in I$ such that $\operatorname{var}_{g}[t, s]<\delta$.

Definition 4.11 A set $S \subset \mathrm{BC}_{g}(I, \mathbb{R})$ is said to be $g$-stable if, for every $t \in[a, b) \cap D_{g}$ and every $\varepsilon \in \mathbb{R}^{+}$, there exist $\delta \in \mathbb{R}^{+}$and a finite covering $\left\{X_{k}\right\}_{k=1}^{m}$ of $[t, t+\delta) \cap I$ such that $|f(x)-f(y)|<\varepsilon$ for every $f \in S$, every $x, y \in X_{k}$ and every $k=1, \ldots, m$.

Remark 4.12 Observe that in Definition 4.11 we can change $[t, t+\delta) \cap I$ by $(t, t+\delta) \cap I$. Indeed, if $\left\{X_{k}\right\}_{k=1}^{m}$ is a suitable finite covering of $(t, t+\delta) \cap I$, then $\{t\} \cup\left\{X_{k}\right\}_{k=1}^{m}$ is an appropriate finite covering of $[t, t+\delta) \cap I$.

Stability is a property that serves to compensate the lack of compactness of $\left(I, \tau_{g}\right)$. The space $\left(I, \tau_{g}\right)$ is not compact in general because, as it was pointed out in [7, Example 3.3], there are $g$-continuous functions defined on $I$ which are not bounded, something that would always be the case if $\left(I, \tau_{g}\right)$ was compact. Stability has been present in the literature in several ways. In [7, Theorem 4.2(iii)], for regulated functions, they ask $S$ to have uniform right-hand side limits in $t \in[a, b) \cap D_{g}$, something which coincides with our definition in that case. It also appears in [19, Theorem 1] (named stability as well) or [3, Sect. 2.12, p. 62] (named equiconvergence) with a different formulation consistent with the topology of $\mathbb{R}$.

Lemma 4.13 Let $S \subset \mathrm{BC}_{g}(I, \mathbb{R})$. If $S$ is uniformly g-equicontinuous, then $S$ is g-equicontinuous and $g$-stable.

Proof Clearly, $S$ is $g$-equicontinuous. Take $t \in[a, b) \cap D_{g}$ and fix $\varepsilon \in \mathbb{R}^{+}$. Let $\rho \in \mathbb{R}^{+}$be such that $|f(x)-f(y)|<\varepsilon$ for every $f \in S$ and every $x, y \in I$ such that $\operatorname{var}_{g}[x, y]<\rho$. Now, there exists $\delta \in \mathbb{R}^{+}$such that $\operatorname{var}_{g}(t, t+\delta)<\rho$ because $g$ is regulated. Thus, by Remark 4.12, $S$ is $g$-stable.

Theorem 4.14 Let $S \subset \mathrm{BC}_{g}(I, \mathbb{R})$, then $S$ is precompact if and only if

(i) $S(t)$ is bounded for all $t \in I$;

(ii) $S$ is g-equicontinuous;

(iii) $S$ is $g$-stable.

Proof As we have argued before, $S(t)$ is precompact if and only if $S(t)$ is bounded, so Conditions (i) in both Theorem 4.9 and Theorem 4.14 coincide.

Now, let us see that, if (i)-(iii) hold, then $S$ satisfies Condition (ii) of Theorem 4.9. Fix $\varepsilon \in \mathbb{R}^{+}$. For every $t \in I$, there exists $\delta_{t} \in \mathbb{R}^{+}$such that $|f(t)-f(s)|<\frac{\varepsilon}{2}$ for every $f \in S$ and every $s \in I$ such that $\operatorname{var}_{g}[t, s]<\delta_{t}$. Consider $U_{t}:=B_{g}\left(t, \delta_{t}\right)$ for every $t \in I$. If $t \in I \backslash D_{g}$, then, 
by the continuity of $g$ at $t$, there exists $\rho_{t} \in \mathbb{R}^{+}$such that $V_{t}=\left(t-\rho_{t}, t+\rho_{t}\right) \cap I \subset U_{t}$. On the other hand, if $t \in D_{g}$, since $g$ is left continuous, there exists $\sigma_{t} \in \mathbb{R}^{+}$such that $\left(t-\sigma_{t}, t\right] \cap I \subset U_{t} \cap[a, t]$. Finally, since $S$ is $g$-stable, there exists $\eta_{t} \in \mathbb{R}^{+}$and a finite covering $\left\{X_{t, k}\right\}_{k=1}^{m_{t}}$ of $\left[t, t+\eta_{t}\right) \cap I$ such that $|f(x)-f(y)|<\varepsilon$ for every $f \in S$, every $x, y \in X_{t, k}$ and every $k=1, \ldots, m_{t}$. For $t \in D_{g}$, we set $\rho_{t}:=\min \left\{\sigma_{t}, \eta_{t}\right\}$. We denote $V_{t}:=\left(t-\rho_{t}, t+\rho_{t}\right) \cap I$. The set $\left\{V_{t}\right\}_{t \in I}$ is an open cover of $\left(I, \tau_{u}\right)$, so there exists a finite subcover $\left\{V_{t_{i}}\right\}_{i=1}^{j}$.

We consider the finite covering of $I$,

$$
\begin{aligned}
\mathcal{Y}:= & \left\{V_{t_{i}}: i \in\{1, \ldots, j\} \text { and } t_{i} \notin D_{g}\right\} \cup\left\{V_{t_{i}} \backslash\left[t_{i}, t_{i}+\rho_{t_{i}}\right): i \in\{1, \ldots, j\} \text { and } t_{i} \in D_{g}\right\} \\
& \cup\left\{\left[t_{i}, t_{i}+\rho_{t_{i}}\right) \cap X_{t_{i}, k_{i}}: i \in\{1, \ldots, j\}, t_{i} \in D_{g} \text { and } k_{i} \in\left\{i, \ldots, m_{t_{i}}\right\}\right\} .
\end{aligned}
$$

If $x, y \in V_{t_{i}}$ for some $t_{i} \notin D_{g}$ or if $x, y \in V_{t_{i}} \backslash\left[t_{i}, t_{i}+\rho_{t_{i}}\right)$ for some $t_{i} \in D_{g}$, then $x, y \in U_{t_{i}}$ and

$$
|f(x)-f(y)| \leq\left|f(x)-f\left(t_{i}\right)\right|+\left|f\left(t_{i}\right)-f(y)\right|<\varepsilon \quad \text { for every } f \in S .
$$

Also, if $x, y \in\left[t_{i}, t_{i}+\rho_{t_{i}}\right) \cap X_{t_{i}, k_{i}}$ for some $t_{i} \in D_{g}$ and some $k_{i} \in\left\{1, \ldots, m_{t_{i}}\right\}$, then $|f(x)-f(y)|<$ $\varepsilon$ for every $f \in S$. Therefore, $S$ satisfies (ii) of Theorem 4.9 and hence, $S$ is precompact.

Now, let us assume that $S$ is precompact. It was noticed before that $S$ satisfies (i). Let $\varepsilon>0$. For $t \notin D_{g}$ and for $\rho>0$, we consider the set

$$
S_{\varepsilon, t}(\rho):=\left\{f \in S:|f(t)-f(s)|<\varepsilon \text { for all } s \in I \text { such that } \operatorname{var}_{g}[t, s]<\rho\right\} .
$$

It is easy to see that $\left\{S_{\varepsilon, t}(\rho)\right\}_{\rho \in \mathbb{R}^{+}}$is an open cover of $\bar{S}$. Since $S$ is precompact, and $\mathrm{BC}_{g}(I, \mathbb{R})$ is complete, $\bar{S}$ is compact, so there is a finite subcover $\left\{S_{\varepsilon, t}\left(\rho_{k}\right)\right\}_{k=1, \ldots, m}$. Take $\delta:=\min \left\{\rho_{k}: k=1, \ldots, m\right\}$. Then, if $s \in I$ is such that $\operatorname{var}_{g}[t, s]<\delta$, one has $|f(t)-f(s)|<\varepsilon$ for every $f \in S$. Similarly, for $t \in D_{g}$, using the left continuity of $f \in S$ at $t$, one can see that $\left\{S_{\varepsilon, t}(\rho)\right\}_{\rho \in\left(0, g\left(t^{+}\right)-g(t)\right)}$ is an open cover of $\bar{S}$. Again, the precompactness of $S$ ensures the existence of a finite subcover $\left\{S_{\varepsilon, t}\left(\rho_{k}\right)\right\}_{k=1, \ldots, m}$. Thus, if $s \in I$ is such that $\operatorname{var}_{g}[t, s]<\min \left\{\rho_{k}: k=\right.$ $1, \ldots, m\}$, one has $|f(t)-f(s)|<\varepsilon$ for every $f \in S$. So, $S$ is $g$-equicontinuous. Finally, Theorem 4.9 implies that there exists a finite covering $\left\{X_{k}\right\}_{k=1}^{m}$ of $I$ such that $|f(x)-f(y)|<\varepsilon$ for every $f \in S$, every $x, y \in X_{k}$ and every $k \in\{1, \ldots, m\}$. Therefore $S$ is $g$-stable.

Combining Lemma 4.13 and Theorem 4.14, we deduce the following result.

Corollary 4.15 Let $S \subset \mathrm{BC}_{g}(I, \mathbb{R})$. If

(i) $S(t)$ is bounded for all $t \in I$ and

(ii) $S$ is uniformly g-equicontinuous,

then $S$ is precompact.

\section{The $g$-derivative}

We now generalize to nonmonotonic derivator $g$ the notion of $g$-derivative introduced in [14].

Definition 5.1 The derivative with respect to $g$ (or $g$-derivative) of a function $f: I \rightarrow \mathbb{R}$ at a point $x \in D_{g} \cup\left(I \backslash\left(F_{g} \cup \overline{C_{g}}\right)\right)$ is defined as follows, provided that the corresponding limits 
exist:

$$
f_{g}^{\prime}(x)= \begin{cases}\lim _{y \rightarrow x} \frac{f(y)-f(x)}{g(y)-g(x)} & \text { if } x \notin D_{g}, \\ \lim _{y \rightarrow x^{+}} \frac{f(y)-f(x)}{g(y)-g(x)} & \text { if } x \in D_{g} .\end{cases}
$$

Proposition 5.2 Let $f: I \rightarrow \mathbb{R}$ and $x_{0} \in D_{g} \cup\left(I \backslash\left(F_{g} \cup \overline{C_{g}}\right)\right)$. Then the following statements are equivalent:

(1) The map $f$ is $g$-differentiable at $x_{0}$.

(2) There exist $\alpha \in \mathbb{R}$, a set $N \subset I$ containing $x_{0}$ and a function $\varphi: N \rightarrow \mathbb{R}$ such that $\varphi\left(x_{0}\right)=0, g(x) \neq g\left(x_{0}\right)$ for every $x \in N \backslash\left\{x_{0}\right\}$,

$$
f(x)=f\left(x_{0}\right)+[\varphi(x)+\alpha]\left[g(x)-g\left(x_{0}\right)\right] \text { for every } x \in N,
$$

and one of the following holds:

(i) $x_{0} \notin D_{g}, N$ is open in $I$ and $\varphi$ is continuous at $x_{0}$;

(ii) $x_{0} \in D_{g}, N$ is open in $\left[x_{0}, \infty\right) \cap I$ and $\varphi$ is right continuous at $x_{0}$.

In the case (2), we have that $\alpha=f_{g}^{\prime}\left(x_{0}\right)$.

Proof $(1) \Rightarrow$ (2) If $f$ is $g$-differentiable at $x_{0} \notin D_{g}$ (resp. $x_{0} \in D_{g}$ ), then $g(x) \neq g\left(x_{0}\right)$ in a neighborhood $N$ of $x_{0}$ in $I$ (resp. in $I \cap\left[x_{0}, \infty\right)$ ) (otherwise we could not take the limit that defines $\left.f_{g}^{\prime}\left(x_{0}\right)\right)$. Hence, it is enough to define

$$
\varphi(x):=\frac{f(x)-f\left(x_{0}\right)}{g(x)-g\left(x_{0}\right)}-f_{g}^{\prime}\left(x_{0}\right)
$$

for $x \in N \backslash\left\{x_{0}\right\}$ and $\varphi\left(x_{0}\right)=0$. By the definition of the derivative, $\varphi$ is continuous at $x_{0}$ (resp. right continuous at $\left.x_{0}\right)$.

$(2) \Rightarrow(1)$ Since $\varphi$ is right continuous at $x_{0}$,

$$
\lim _{x \rightarrow x_{0}^{+}} \frac{f(x)-f\left(x_{0}\right)}{g(x)-g\left(x_{0}\right)}=\lim _{x \rightarrow x_{0}^{+}}(\varphi(x)+\alpha)=\alpha .
$$

In the case where $x_{0} \notin D_{g}$, we have the analogous result with the left limit. So, in either case, there exists $f_{g}^{\prime}\left(x_{0}\right)=\alpha$.

Remark 5.3 Now, we see why it is important that $g$ is of controlled variation. Assume, for instance, that we have a point $t_{0} \in(a, b) \backslash D_{g}$ such that there are two sequences $\left\{t_{n}\right\}$, $\left\{s_{n}\right\}$ in $\left(a, t_{0}\right)$ converging to $t_{0}$ and satisfying that $g\left(t_{n}\right)<g\left(t_{0}\right)<g\left(s_{n}\right)$; that is, that $t_{0}$ does not belong to any open interval where $g$ is monotonic. Furthermore, assume that $g$ is differentiable at $t_{0}$. Then, if $f$ is $g$-differentiable at $t_{0}$, we have that

$$
f^{\prime}\left(t_{0}\right)=\lim _{t \rightarrow t_{0}} \frac{f(t)-f\left(t_{0}\right)}{t-t_{0}}=\lim _{t \rightarrow t_{0}} \frac{f(t)-f\left(t_{0}\right)}{g(t)-g\left(t_{0}\right)} \frac{g(t)-g\left(t_{0}\right)}{t-t_{0}}=f_{g}^{\prime}\left(t_{0}\right) g^{\prime}\left(t_{0}\right) .
$$

But also, observe that, for every $n \in \mathbb{N}$,

$$
\frac{g\left(t_{n}\right)-g\left(t_{0}\right)}{t_{n}-t_{0}}>0>\frac{g\left(s_{n}\right)-g\left(t_{0}\right)}{s_{n}-t_{0}},
$$

which implies that $g^{\prime}\left(t_{0}\right)=0$. Hence, $f^{\prime}\left(t_{0}\right)=0$. 
In conclusion, the fact that $t_{0}$ does not belong to any open interval where $g$ is monotonic forces very restrictive conditions on the behavior of $f$.

The next result establishes a more general chain rule than the one in [14].

Proposition 5.4 (Chain rule) Let $g_{1} \in \mathrm{CV}_{-}((a, b), \mathbb{R}), g_{2} \in \mathrm{CV}_{-}((c, d), \mathbb{R}), f:(a, b) \rightarrow(c, d)$ and $h:(c, d) \rightarrow \mathbb{R}$. Assume that $g_{2} \circ f$ is $g_{1}$-differentiable at some $x_{0} \in(a, b)$ with $f\left(x_{0}\right) \notin D_{g_{2}}$, and $h$ is $g_{2}$-differentiable at $f\left(x_{0}\right)$. Then, $h \circ f$ is $g_{1}$-differentiable at $x_{0}$ and

$$
(h \circ f)_{g_{1}}^{\prime}\left(x_{0}\right)=h_{g_{2}}^{\prime}\left(f\left(x_{0}\right)\right)\left(g_{2} \circ f\right)_{g_{1}}^{\prime}\left(x_{0}\right) .
$$

Proof We write the proof for $x_{0} \notin D_{g_{1}}$. It is analogous for $x_{0} \in D_{g_{1}}$. Observe that, since $\left(g_{2} \circ f\right)_{g_{1}}^{\prime}$ exists, $g_{1}(x) \neq g_{1}\left(x_{0}\right)$ in a neighborhood of $x_{0}$. Since $h$ is $g_{2}$-differentiable at $f\left(x_{0}\right) \notin$ $D_{g_{2}}$, by Proposition 5.2, there exist $N$ a neighborhood of $f\left(x_{0}\right)$ and a function $\varphi: N \rightarrow \mathbb{R}$ continuous at $f\left(x_{0}\right)$ and such that $\varphi\left(f\left(x_{0}\right)\right)=0$ and

$$
h(y)=h\left(f\left(x_{0}\right)\right)+\left[\varphi(y)+h_{g_{2}}^{\prime}\left(f\left(x_{0}\right)\right)\right]\left[g_{2}(y)-g_{2}\left(f\left(x_{0}\right)\right)\right] .
$$

Therefore,

$$
\frac{h(f(x))-h\left(f\left(x_{0}\right)\right)}{g_{1}(x)-g_{1}\left(x_{0}\right)}=\left(\varphi(f(x))+h_{g_{2}}^{\prime}\left(f\left(x_{0}\right)\right)\right) \frac{g_{2}(f(x))-g_{2}\left(f\left(x_{0}\right)\right)}{g_{1}(x)-g_{1}\left(x_{0}\right)} .
$$

Taking the limit, we have that

$$
(h \circ f)_{g_{1}}^{\prime}\left(x_{0}\right)=h_{g_{2}}^{\prime}\left(f\left(x_{0}\right)\right)\left(g_{2} \circ f\right)_{g_{1}}^{\prime}\left(x_{0}\right) .
$$

The following corollary is obtained by taking $g_{2}$ as the identity.

Corollary 5.5 Let $g \in \mathrm{CV}_{-}(I, \mathbb{R}), f: I \rightarrow(c, d)$ a function $g$-differentiable at $x_{0}$ and $h$ : $(c, d) \rightarrow \mathbb{R}$ a function differentiable at $f\left(x_{0}\right)$. Then, $(h \circ f)$ is $g$-differentiable at $x_{0}$ and $(h \circ f)_{g}^{\prime}\left(x_{0}\right)=h^{\prime}\left(f\left(x_{0}\right)\right) f_{g}^{\prime}\left(x_{0}\right)$.

\section{The fundamental theorem of calculus}

From now on, we consider $g \in \mathrm{CV}_{-}(I, \mathbb{R})$ and the sets associated to $g$ provided by Definition 3.7,

$$
A_{g}^{+}=\bigcup_{\lambda^{+} \in \Lambda^{+}} I_{\lambda^{+}} \quad \text { and } \quad A_{g}^{-}=\bigcup_{\lambda^{-} \in \Lambda^{-}} I_{\lambda^{-}} .
$$

Definition 6.1 A function $h: I \rightarrow \mathbb{R}$ is said to be $g$-absolutely continuous if it is $g$ continuous and if, for every $\varepsilon \in \mathbb{R}^{+}$, there exists $\delta \in \mathbb{R}^{+}$such that, for any family $\left\{\left(\alpha_{\xi}, \beta_{\xi}\right)\right\}_{\xi \in \Xi^{+}}$of pairwise disjoint subintervals of $A_{g}^{+}$, any family $\left\{\left(\alpha_{\xi}, \beta_{\xi}\right)\right\}_{\xi \in \Xi^{-}}$of pairwise disjoint subintervals of $A_{g}^{-}$, and any sets $E^{+} \subset D_{g}^{+}, E^{-} \subset D_{g}^{-}$, we have that, if

$$
\sum_{\xi \in \Xi^{+}}\left[g\left(\beta_{\xi}\right)-g\left(\alpha_{\xi}^{+}\right)\right]+\sum_{\xi \in \Xi^{-}}\left[g\left(\alpha_{\xi}^{+}\right)-g\left(\beta_{\xi}\right)\right]+\sum_{t \in E^{+}}\left[g\left(t^{+}\right)-g(t)\right]+\sum_{t \in E^{-}}\left[g(t)-g\left(t^{+}\right)\right]<\delta,
$$


then

$$
\sum_{\xi \in \Xi^{+} \cup \Xi^{-}}\left|h\left(\beta_{\xi}\right)-h\left(\alpha_{\xi}^{+}\right)\right|+\sum_{t \in E^{+} \cup E^{-}}\left|h\left(t^{+}\right)-h(t)\right|<\varepsilon
$$

We denote by $\operatorname{AC}_{g}(I, \mathbb{R})$ the set of $g$-absolutely continuous functions.

In the next two theorems, we show that, from $\mu_{g}$-integrable maps, we can defined $g$ absolutely continuous functions which are $g$-differentiable $\left|\mu_{g}\right|$-almost every where.

Theorem 6.2 Let $v \in \mathrm{L}_{g}^{1}([a, b))$ and $h(x):=\int_{[a, x)} v(s) \mathrm{d} \mu_{g}$ for $x \in I$. Then $h \in \operatorname{AC}_{g}(I, \mathbb{R})$.

Proof We consider the case $v \geq 0\left|\mu_{g}\right|$-a.e. on $I$ since $v$ has to be the difference of two such functions $\left|\mu_{g}\right|$-a.e. Let $\varepsilon \in \mathbb{R}^{+}$. Since $v \in \mathrm{L}_{g}^{1}([a, b))$, there exists $\delta \in \mathbb{R}^{+}$such that $\int_{E} v \mathrm{~d}\left|\mu_{g}\right|<$ $\varepsilon$ if $\left|\mu_{g}\right|(E)<\delta$. Then, for $\operatorname{var}_{g}[x, y]=\left|\mu_{g}\right|([x, y))<\delta$, we have that

$$
|h(y)-h(x)|=\left|\int_{[x, y)} v \mathrm{~d} \mu_{g}\right| \leq \int_{E} v \mathrm{~d}\left|\mu_{g}\right|<\varepsilon,
$$

so $h$ is $g$-continuous.

Now, take a family $\left\{\left(\alpha_{\xi}, \beta_{\xi}\right)\right\}_{\xi \in \Xi^{+}}$of pairwise disjoint subintervals of $A_{g}^{+}$, a family $\left\{\left(\alpha_{\xi}, \beta_{\xi}\right)\right\}_{\xi \in \Xi^{-}}$of pairwise disjoint subintervals of $A_{g}^{-}$, and sets $E^{+} \subset D_{g}^{+}, E^{-} \subset D_{g}^{-}$such that

$$
\sum_{\xi \in \Xi^{+}}\left[g\left(\beta_{\xi}\right)-g\left(\alpha_{\xi}^{+}\right)\right]+\sum_{\xi \in \Xi^{-}}\left[g\left(\alpha_{\xi}^{+}\right)-g\left(\beta_{\xi}\right)\right]+\sum_{t \in E^{+}}\left[g\left(t^{+}\right)-g(t)\right]+\sum_{t \in E^{-}}\left[g(t)-g\left(t^{+}\right)\right]<\delta .
$$

Let us denote $B^{+}=\bigcup_{\xi \in \Xi^{+}}\left(\alpha_{\xi}, \beta_{\xi}\right)$ and $B^{-}=\bigcup_{\xi \in \Xi^{-}}\left(\alpha_{\xi}, \beta_{\xi}\right)$. Observe that the sets $B^{+}, B^{-}$, $E^{+}$and $E^{-}$are pairwise disjoint, so

$$
\left|\mu_{g}\right|\left(B^{+} \cup B^{-} \cup E^{+} \cup E^{-}\right)=\left|\mu_{g}\right|\left(B^{+}\right)+\left|\mu_{g}\right|\left(B^{-}\right)+\left|\mu_{g}\right|\left(E^{+}\right)+\left|\mu_{g}\right|\left(E^{-}\right) .
$$

Furthermore, since $g$ is nondecreasing on the connected components of $B^{+}$, we have that $\left|\mu_{g}\right|\left(B^{+}\right)=\mu_{g}^{+}\left(B^{+}\right)$. By similar observations we get that

$$
\left|\mu_{g}\right|\left(B^{+} \cup B^{-} \cup E^{+} \cup E^{-}\right)=\mu_{g}^{+}\left(B^{+}\right)+\mu_{g}^{-}\left(B^{-}\right)+\mu_{g}^{+}\left(E^{+}\right)+\mu_{g}^{-}\left(E^{-}\right) .
$$

Because $g$ is left continuous, the measure in each of the intervals $\left(\alpha_{\xi}, \beta_{\xi}\right)$ is given by $\mid g\left(\beta_{\xi}\right)-$ $g\left(\alpha_{\xi}^{+}\right) \mid$, so we have that

$$
\begin{aligned}
& \left|\mu_{g}\right|\left(B^{+} \cup B^{-} \cup E^{+} \cup E^{-}\right)=\mu_{g}^{+}\left(B^{+}\right)+\mu_{g}^{-}\left(B^{-}\right)+\mu_{g}^{+}\left(E^{+}\right)+\mu_{g}^{-}\left(E^{-}\right) \\
& =\sum_{\xi \in \Xi^{+}}\left[g\left(\beta_{\xi}\right)-g\left(\alpha_{\xi}^{+}\right)\right]-\sum_{\xi \in \Xi^{-}}\left[g\left(\beta_{\xi}\right)-g\left(\alpha_{\xi}^{+}\right)\right] \\
& \quad+\sum_{t \in E^{+}}\left[g\left(t^{+}\right)-g(t)\right]-\sum_{t \in E^{-}}\left[g\left(t^{+}\right)-g(t)\right] \\
& \quad<\delta .
\end{aligned}
$$


Thus,

$$
\sum_{\xi \in \Xi^{+} \cup \Xi^{-}}\left|h\left(\beta_{\xi}\right)-h\left(\alpha_{\xi}^{+}\right)\right|+\sum_{t \in E^{+} \cup E^{-}}\left|h\left(t^{+}\right)-h(t)\right|=\int_{B^{+} \cup B^{-}} v \mathrm{~d}\left|\mu_{g}\right|+\int_{E^{+} \cup E^{-}} v \mathrm{~d}\left|\mu_{g}\right|<\varepsilon .
$$

Hence, $h \in \operatorname{AC}_{g}(I, \mathbb{R})$.

Theorem 6.3 Let $v \in \mathrm{L}_{g}^{1}([a, b))$ and $h(x):=\int_{[a, x)} v \mathrm{~d} \mu_{g}$ for $x \in I$. Then, $h_{g}^{\prime}=v\left|\mu_{g}\right|$-a.e. on $I$.

Proof For the proof, we use [14, Theorem 2.4], a theorem with the same statement but for a nondecreasing $g$. By definition, $A_{g}^{+}=\bigcup_{\lambda \in \Lambda^{+}} I_{\lambda}$ and $\left.g\right|_{I_{\lambda}}$ is nondecreasing for every $\lambda \in \Lambda^{+}$. If we consider $h_{\lambda}(x)=\int_{\left[a_{\lambda}, x\right)} v \mathrm{~d} \mu_{\tilde{g}}$, where $\widetilde{g}(s)=g(s)$ for $s \in\left(a_{\lambda}, b\right]$ and $\widetilde{g}\left(a_{\lambda}\right):=g\left(a_{\lambda}^{+}\right)$, we have

$$
h_{\lambda}(x)=\int_{\left[a_{\lambda}, x\right)} v \mathrm{~d} \mu_{\widetilde{g}}=\int_{\left[a_{\lambda}, x\right)} v \mathrm{~d}\left|\mu_{\tilde{g}}\right|
$$

It follows from [14, Theorem 2.4] that $h_{g}^{\prime}=\left(h_{\lambda}\right)_{g}^{\prime}=\left(h_{\lambda}\right)_{\widetilde{g}}^{\prime}=v$ holds $\left|\mu_{g}\right|$-a.e. on $I_{\lambda}$. Since $\Lambda^{+}$ is countable, we deduce that

$$
h_{g}^{\prime}=v \quad\left|\mu_{g}\right| \text {-a.e. on } A_{g}^{+} .
$$

Analogously, $A_{g}^{-}=\bigcup_{\lambda \in \Lambda^{-}} I_{\lambda}$ and $-\left.g\right|_{I_{\lambda}}$ is nondecreasing for every $\lambda \in \Lambda^{-}$. So, for $x \in I_{\lambda}$,

$$
h_{\lambda}(x)=\int_{\left[a_{\lambda}, x\right)} v \mathrm{~d}\left(-\mu_{\tilde{g}}^{\bar{g}}\right)=-\int_{\left[a_{\lambda}, x\right)} v \mathrm{~d} \mu_{-\widetilde{g}}
$$

and

$$
h_{g}^{\prime}=v \quad\left|\mu_{g}\right| \text {-a.e. on } A_{g}^{-} \text {. }
$$

Let $t \in D_{g}$. Then

$$
h_{g}^{\prime}(x)=\frac{h\left(x^{+}\right)-h(x)}{g\left(x^{+}\right)-g(x)}=\frac{\int_{\left[x, x^{+}\right)} v \mathrm{~d} \mu_{g}}{g\left(x^{+}\right)-g(x)}=\frac{v(x)\left(g\left(x^{+}\right)-g(x)\right)}{g\left(x^{+}\right)-g(x)}=v(x) .
$$

Hence, $h_{g}^{\prime}=v$ in $D_{g}$.

Finally, $h_{g}^{\prime}=v\left|\mu_{g}\right|$-a.e. on $I$ since $I \backslash\left(A_{g}^{+} \cup A_{g}^{-} \cup D_{g}\right)=F_{g} \backslash D_{g}$ and $\left|\mu_{g}\right|\left(F_{g} \backslash D_{g}\right)=0$.

Now, we show that $g$-absolutely continuous maps have bounded variation.

Theorem 6.4 If $h \in \mathrm{AC}_{g}(I, \mathbb{R})$, then $h \in \mathrm{BV}_{-}(I, \mathbb{R})$.

Proof By definition, $A_{g}^{+}=\bigcup_{\lambda \in \Lambda^{+}} I_{\lambda}, A_{g}^{-}=\bigcup_{\lambda \in \Lambda^{-}} I_{\lambda}$ with $I_{\lambda}=\left(a_{\lambda}, b_{\lambda}\right)$. Observe that, in general, for $\alpha \leq \beta \leq \gamma, \operatorname{var}_{h}[\alpha, \gamma]=\operatorname{var}_{h}[\alpha, \beta]+\operatorname{var}_{h}[\beta, \gamma]$. Hence, in order to bound the variation on $I$, we realize that it has to be less or equal than the sum of the variation in the intervals $\left(a_{\lambda}, b_{\lambda}\right]$ for $\lambda \in \Lambda^{+} \cup \Lambda^{-}$plus the jumps at the points of $D_{g}$ plus an extra term 
where we make up for the fact that we have not considered the closed sets $\left[a_{\lambda}, b_{\lambda}\right]$, but omitted the point $a_{\lambda}$. Taking this into account, we have that

$$
\begin{aligned}
\operatorname{var}_{h} I & \leq \sum_{\lambda \in \Lambda^{+} \cup \Lambda^{-}} \operatorname{var}_{h}\left(a_{\lambda}, b_{\lambda}\right]+\sum_{\lambda \in \Lambda^{+} \cup \Lambda^{-}}\left|h\left(a_{\lambda}^{+}\right)-h\left(a_{\lambda}\right)\right|+\sum_{t \in D g}\left|h\left(t^{+}\right)-h(t)\right| \\
& \leq \sum_{\lambda \in \Lambda^{+} \cup \Lambda^{-}} \operatorname{var}_{h}\left(a_{\lambda}, b_{\lambda}\right]+\sum_{t \in D_{h}}\left|h\left(t^{+}\right)-h(t)\right| .
\end{aligned}
$$

For $\varepsilon=1$, let $\delta \in \mathbb{R}^{+}$be given in Definition 6.1.

First, let us prove that $\sum_{t \in D_{h}}\left|h\left(t^{+}\right)-h(t)\right|<\infty$. By Proposition 4.7, $D_{h} \subset D_{g}$. Since $D_{g}$ is at most countable, without loss of generality, one can write $D_{g}=\left\{t_{n}: n \in \mathbb{N}\right\}$. Since $g \in \mathrm{CV}_{-}(I, \mathbb{R})$, we can fix $N_{0} \in \mathbb{N}$ such that $\sum_{n=N_{0}+1}^{\infty}\left|g\left(t_{n}^{+}\right)-g\left(t_{n}\right)\right|<\delta$. Therefore, $\sum_{n=N_{0}+1}^{\infty}\left|h\left(t_{n}^{+}\right)-h\left(t_{n}\right)\right|<1$. So,

$$
\sum_{t \in D_{h}}\left|h\left(t^{+}\right)-h(t)\right|<1+\sum_{n=1}^{N_{0}}\left|h\left(t_{n}^{+}\right)-h\left(t_{n}\right)\right|<\infty
$$

Now, let us prove that

$$
\sum_{\lambda \in \Lambda^{+} \cup \Lambda^{-}} \operatorname{var}_{h}\left(a_{\lambda}, b_{\lambda}\right]<\infty
$$

The set $\Lambda^{+} \cup \Lambda^{-}$is at most countable and, without lost of generality, it can be written $\left\{\lambda_{n}: n \in \mathbb{N}\right\}$. Since $g$ has bounded variation, there exists $N \in \mathbb{N}$ such that

$$
\sum_{n=N+1}^{\infty}\left|g\left(b_{\lambda_{n}}\right)-g\left(a_{\lambda_{n}}\right)\right|<\delta
$$

For every $n>N$ and every $\left\{x_{0}^{n}, \ldots, x_{m_{n}}^{n}\right\} \in \mathcal{P}\left(a_{\lambda_{n}}, b_{\lambda_{n}}\right)$, using the fact that $g$ is monotonic on $\left(a_{\lambda_{n}}, b_{\lambda_{n}}\right]$, we get

$$
\sum_{n=N+1}^{\infty} \sum_{i=1}^{m_{n}}\left|g\left(x_{i}^{n}\right)-g\left(x_{i-1}^{n}\right)\right|=\sum_{n=N+1}^{\infty}\left|g\left(x_{m_{n}}^{n}\right)-g\left(x_{0}^{n}\right)\right| \leq \sum_{n=N+1}^{\infty}\left|g\left(b_{\lambda_{n}}\right)-g\left(a_{\lambda_{n}}\right)\right|<\delta .
$$

Thus,

$$
\sum_{n=N+1}^{\infty} \sum_{i=1}^{m_{n}}\left|h\left(x_{i}^{n}\right)-h\left(x_{i-1}^{n}\right)\right|<1,
$$

and hence

$$
\sum_{n=N+1}^{\infty} \operatorname{var}_{h}\left(a_{\lambda_{n}}, b_{\lambda_{n}}\right] \leq 1
$$

Therefore, for $n=1, \ldots, N$, since $g$ is monotonic on $\left(a_{\lambda_{n}}, b_{\lambda_{n}}\right.$ ], arguing as in [14, Proposition 5.3], we deduce that

$$
\operatorname{var}_{h}\left(a_{\lambda_{n}}, b_{\lambda_{n}}\right]<\infty
$$


Combining (6.1)-(6.4), we conclude that $\operatorname{var}_{h} I<\infty$. Finally, since $h$ is $g$-continuous, it is left continuous. Therefore, $h \in \mathrm{BV}_{-}(I, \mathbb{R})$.

Remark 6.5 For $h \in \mathrm{AC}_{g}(I, \mathbb{R})$, since $h \in \mathrm{BV}_{-}(I, \mathbb{R})$ by the previous theorem, we can define the measures $\mu_{h}$ and $\left|\mu_{h}\right|$ associated to $h$ as we did in the preliminaries.

Lemma 6.6 Let $h: I \rightarrow \mathbb{R}$ be a g-absolutely continuous function and $\mu_{h}$ its associated measure. Then, $\mu_{h}$ is $\mu_{g}^{+}$-absolutely continuous on $A_{g}^{+}$(that is, $\mu_{g}^{+}(E)=0$ implies that $\mu_{h}(E)=0$ for any $\mu_{g}^{+}$-measurable set $\left.E \subset A_{g}^{+}\right)$and $\mu_{h}$ is $\mu_{g}^{-}$-absolutely continuous on $A_{g}^{-}$.

Proof We first show that, for any $\varepsilon \in \mathbb{R}^{+}$, there exists $\delta \in \mathbb{R}^{+}$such that, for any open set $V \subset A_{g}^{+}$satisfying $\left|\mu_{g}^{+}(V)\right|<\delta$, we have that $\left|\mu_{h}(V)\right| \leq \varepsilon$. Fix an open set $V \subset A_{g}^{+}$such that $\left|\mu_{g}^{+}(V)\right|<\delta$. The set $V$ can be written as a countable pairwise disjoint union of the form

$$
V=\bigcup_{\zeta \in Z^{+}}\left(r_{\zeta}, s_{\zeta}\right), \quad \text { where }\left(r_{\zeta}, s_{\zeta}\right) \subset I_{\lambda} \text { for some } \lambda \in \Lambda^{+} .
$$

Take $\tilde{r}_{\zeta} \in\left(r_{\zeta}, s_{\zeta}\right)$ for every $\zeta \in Z^{+}$. Hence,

$$
\sum_{\zeta \in Z^{+}}\left[g\left(s_{\zeta}\right)-g\left(\widetilde{r}_{\zeta}\right)\right]=\mu_{g}\left(\bigcup_{\zeta \in Z^{+}}\left[\widetilde{r}_{\zeta}, s_{\zeta}\right)\right) \leq \mu_{g}^{+}(V)<\delta,
$$

and, arguing as in the proof of the previous theorem, we get

$$
\left.\sum_{\zeta \in Z^{+}} \operatorname{var}_{h}\left(\widetilde{r}_{\zeta}, s_{\zeta}\right)\right) \leq \varepsilon
$$

Letting $\widetilde{r}_{\zeta} \rightarrow r_{\zeta}$, we obtain

$$
\left|\mu_{h}\right|(V)=\sum_{\zeta \in Z^{+}} \operatorname{var}_{h}\left(\left(r_{\zeta}, s_{\zeta}\right)\right) \leq \varepsilon
$$

Now, since $\mu_{g}^{+}$and $\left|\mu_{h}\right|$ are outer regular, for any $\mu_{g}^{+}$-measurable set $E \subset A_{g}^{+}$, there exist open sets $V_{n} \subset A_{g}^{+}$such that $E \subset V_{n}$ for every $n \in \mathbb{N}$ and

$$
\lim _{n \rightarrow \infty} \mu_{g}^{+}\left(V_{n}\right)=\mu_{g}^{+}(E) \quad \text { and } \quad \lim _{n \rightarrow \infty}\left|\mu_{h}\right|\left(V_{n}\right)=\left|\mu_{h}\right|(E) .
$$

Now, for $E \subset A_{g}^{+}$such that $\mu_{g}^{+}(E)=0$, one has $\mu_{g}^{+}\left(V_{n}\right) \rightarrow 0$ and, by the first part of the proof, $\lim _{n \rightarrow \infty}\left|\mu_{h}\right|\left(V_{n}\right)=0$. Therefore, $\left|\mu_{h}\right|(E)=0$ and hence, $\mu_{h}(E)=0$.

Similarly, it can be shown that $\mu_{h}$ is $\mu_{g}^{-}$-absolutely continuous on $A_{g}^{-}$.

We are ready to establish a Fundamental Theorem of Calculus for $g$-absolutely continuous maps.

Theorem 6.7 (Fundamental Theorem of Calculus for the Lebesgue-Stieltjes integral) Let $g \in \mathrm{CV}_{-}(I, \mathbb{R})$ and $h: I \rightarrow \mathbb{R}$. The following statements are equivalent:

(1) $h \in \operatorname{AC}_{g}(I, \mathbb{R})$;

(2) $h$ satisfies the following: 
(a) there exists $h_{g}^{\prime}\left|\mu_{g}\right|$-a.e. in $I$,

(b) $h_{g}^{\prime} \in \mathrm{L}_{g}^{1}(I, \mathbb{R})$,

(c) for each $t \in I$, we have that

$$
h(t)=h(a)+\int_{[a, t)} h_{g}^{\prime} \mathrm{d} \mu_{g}
$$

Proof $(1) \Rightarrow$ (2) We know from Lemma 6.6 that $\mu_{h}$ is $\mu_{g}^{+}$-absolutely continuous on $A_{g}^{+}$. Hence, by the Radon-Nikodym Theorem [1, Theorem 5.3.2], there exists a unique function $v^{+} \in \mathrm{L}_{\mu_{g}^{+}}^{1}\left(A_{g}^{+}\right)$such that

$$
\mu_{h}(E)=\int_{E} v^{+} \mathrm{d} \mu_{g}^{+} \quad \text { for any } \mu_{g}^{+} \text {-measurable set } E \subset A_{g}^{+}
$$

Similarly, there exists a unique function $v^{-} \in \mathrm{L}_{\mu_{g}^{-}}^{1}\left(A_{g}^{-}\right)$such that

$$
\mu_{h}(E)=\int_{E} \nu^{-} \mathrm{d} \mu_{g}^{-} \quad \text { for any } \mu_{g}^{-} \text {-measurable set } E \subset A_{g}^{-} .
$$

Let us define $v: I \rightarrow \mathbb{R}$ by

$$
v(t)= \begin{cases}v^{+}(t) & \text { if } t \in A_{g}^{+}, \\ -v^{-}(t) & \text { if } t \in A_{g}^{-}, \\ \frac{h\left(t^{+}\right)-h(t)}{g\left(t^{+}\right)-g(t)} & \text { if } t \in D_{g}, \\ 0 & \text { if } t \in F_{g} \backslash D_{g} .\end{cases}
$$

It follows from Theorem 6.4 that $v \in L_{g}^{1}(I, \mathbb{R})$ since

$$
\begin{aligned}
\int_{I}|v(t)| \mathrm{d}\left|\mu_{g}\right| & =\int_{A_{g}^{+}}\left|v^{+}(t)\right| \mathrm{d} \mu_{g}^{+}+\int_{A_{g}^{-}}\left|v^{-}(t)\right| \mathrm{d} \mu_{g}^{-}+\int_{D_{g}}|v(t)| \mathrm{d}\left|\mu_{g}\right|+\int_{F_{g} \backslash D_{g}}|v(t)| \mathrm{d}\left|\mu_{g}\right| \\
& =\int_{A_{g}^{+}}\left|v^{+}(t)\right| \mathrm{d} \mu_{g}^{+}+\int_{A_{g}^{-}}\left|v^{-}(t)\right| \mathrm{d} \mu_{g}^{-}+\sum_{t \in D_{g}}\left|h\left(t^{+}\right)-h(t)\right| \\
& <\infty
\end{aligned}
$$

Moreover, for every $E \subset I \mu_{g}$-measurable,

$$
\begin{aligned}
\mu_{h}(E) & =\mu_{h}\left(E \cap A_{g}^{+}\right)+\mu_{h}\left(E \cap A_{g}^{-}\right)+\mu_{h}\left(E \cap D_{g}\right)+\mu_{h}\left(E \cap\left(F_{g} \backslash D_{g}\right)\right) \\
& =\int_{E \cap A_{g}^{+}} v^{+}(t) \mathrm{d} \mu_{g}^{+}-\int_{E \cap A_{g}^{-}}-v^{-}(t) \mathrm{d} \mu_{g}^{-}+\sum_{t \in E \cap D_{g}}\left(h\left(t^{+}\right)-h(t)\right) \\
& =\int_{E \cap A_{g}^{+}} v(t) \mathrm{d} \mu_{g}+\int_{E \cap A_{g}^{-}} v(t) \mathrm{d} \mu_{g}+\int_{E \cap D_{g}} v(t) \mathrm{d} \mu_{g} \\
& =\int_{E} v(t) \mathrm{d} \mu_{g} .
\end{aligned}
$$


In particular, for $E=[a, x)$, with $x \in[a, b]$ we have that

$$
h(x)-h(a)=\mu_{h}([a, x))=\int_{[a, x)} v \mathrm{~d} \mu_{g} .
$$

Finally, by Theorem 6.3, $h_{g}^{\prime}=v\left|\mu_{g}\right|$-a.e. on $I$.

$(2) \Rightarrow(1)$ That is a direct consequence of Theorem 6.2.

Here is another property of $g$-absolutely continuous functions.

Lemma 6.8 Let $h \in \operatorname{AC}_{g}(I, \mathbb{R})$ and $\varphi: h(I) \rightarrow \mathbb{R}$ be a Lipschitz function. Then $\varphi \circ h \in$ $\operatorname{AC}_{g}(I, \mathbb{R})$.

Proof Since $\varphi$ is Lipschitz, there exists $L \in \mathbb{R}^{+}$such that $|\varphi(y)-\varphi(x)| \leq L|y-x|$ for every $x, y \in h(I)$. Fix $\varepsilon \in \mathbb{R}^{+}$and consider a family $\left\{\left(\alpha_{\xi}, \beta_{\xi}\right)\right\}_{\xi \in \Xi^{+}}$of pairwise disjoint subintervals of $A_{g}^{+}$, a family $\left\{\left(\alpha_{\xi}, \beta_{\xi}\right)\right\}_{\xi \in \Xi^{-}}$of pairwise disjoint subintervals of $A_{g}^{-}$, and some sets $E^{+} \subset$ $D_{g}^{+}, E^{-} \subset D_{g}^{-}$. Since $h \in \operatorname{AC}_{g}(I, \mathbb{R}), h$ is $g$-continuous and there exists $\delta \in \mathbb{R}^{+}$such that if

$$
\sum_{\xi \in \Xi^{+}}\left[g\left(\beta_{\xi}\right)-g\left(\alpha_{\xi}^{+}\right)\right]+\sum_{\xi \in \Xi^{-}}\left[g\left(\alpha_{\xi}^{+}\right)-g\left(\beta_{\xi}\right)\right]+\sum_{t \in E^{+}}\left[g\left(t^{+}\right)-g(t)\right]+\sum_{t \in E^{-}}\left[g(t)-g\left(t^{+}\right)\right]<\delta,
$$

then

$$
\sum_{\xi \in \Xi^{+} \cup \Xi^{-}}\left|h\left(\beta_{\xi}\right)-h\left(\alpha_{\xi}^{+}\right)\right|+\sum_{t \in E^{+} \cup E^{-}}\left|h\left(t^{+}\right)-h(t)\right|<\frac{\varepsilon}{L} .
$$

Thus,

$$
\begin{aligned}
& \sum_{\xi \in \Xi^{+} \cup \Xi^{-}}\left|\varphi \circ h\left(\beta_{\xi}\right)-\varphi \circ h\left(\alpha_{\xi}^{+}\right)\right|+\sum_{t \in E^{+} \cup E^{-}}\left|\varphi \circ h\left(t^{+}\right)-\varphi \circ h(t)\right| \\
& \quad \leq L\left(\sum_{\xi \in \Xi^{+} \cup \Xi^{-}}\left|h\left(\beta_{\xi}\right)-h\left(\alpha_{\xi}^{+}\right)\right|+\sum_{t \in E^{+} \cup E^{-}}\left|h\left(t^{+}\right)-h(t)\right|\right)<\varepsilon .
\end{aligned}
$$

We give an example of a $g$-absolutely continuous map with $g$ nonmonotonic which can be obtained from a solution of a system of differential equations with nondecreasing derivators.

Example 6.9 With this example, we show that a $g$-differential equation with a nonmonotonic $g$ can be obtained from systems of differential equations with nondecreasing derivators.

Let $p, q: I \rightarrow \mathbb{R}$ be left continuous and nondecreasing. Assume $p$ is $q$-absolutely continuous. Consider a system of differential equations of the form

$$
\begin{aligned}
& x_{p}^{\prime}(t)=f_{1}(t, x(t), y(t)), \\
& y_{q}^{\prime}(t)=f_{2}(t, x(t), y(t)),
\end{aligned}
$$


for $t \in I$ and suppose it has a solution $(u, v)$. By assumption, $u$ is a solution of the differential equation

$$
x_{p}^{\prime}(t)=f_{1}(t, x(t), v(t))
$$

and $u$ is $p$-absolutely continuous. That implies that $D_{u} \subset D_{p} \subset D_{q}$. Also, since $v$ is $q$ absolutely continuous, $D_{v} \subset D_{q}$. Let us assume that $D_{v}=D_{p}=D_{q}, v \in \mathrm{CV}_{-}(I, \mathbb{R})$, and $v$ and $v_{q}^{\prime}$ are nonzero $\left|\mu_{v}\right|$-a.e. Let us point out that in general $v$ is not monotone. Now, we can consider the following $v$-differential equation:

$$
x_{v}^{\prime}(t)=\frac{f_{1}(t, x(t), v(t))}{f_{2}(t, x(t), v(t))} p_{q}^{\prime}(t)
$$

We have that, for $\left|\mu_{v}\right|$-a.e. $t \in I \backslash D_{v}$,

$$
\begin{aligned}
u_{v}^{\prime}(t) & =\lim _{s \rightarrow t} \frac{u(s)-u(t)}{v(s)-v(t)}=\lim _{s \rightarrow t} \frac{u(s)-u(t)}{p(s)-p(t)} \frac{q(s)-q(t)}{v(s)-v(t)} \frac{p(s)-p(t)}{q(s)-q(t)}=\frac{u_{p}^{\prime}(t)}{v_{q}^{\prime}(t)} p_{q}^{\prime}(t) \\
& =\frac{f_{1}(t, u(t), v(t))}{f_{2}(t, u(t), v(t))} p_{q}^{\prime}(t),
\end{aligned}
$$

and, for $t \in D_{v}$,

$$
\begin{aligned}
u_{v}^{\prime}(t) & =\frac{u\left(t^{+}\right)-u(t)}{v\left(t^{+}\right)-v(t)}=\frac{u\left(t^{+}\right)-u(t)}{p\left(t^{+}\right)-p(t)} \frac{q\left(t^{+}\right)-q(t)}{v(t)-v\left(t^{+}\right)} \frac{p\left(t^{+}\right)-p(t)}{q\left(t^{+}\right)-q(t)}=\frac{u_{p}^{\prime}(t)}{v_{q}^{\prime}(t)} p_{q}^{\prime}(t) \\
& =\frac{f_{1}(t, u(t), v(t))}{f_{2}(t, u(t), v(t))} p_{q}^{\prime}(t) .
\end{aligned}
$$

That is, $u$ is a solution of (6.7) as well.

\section{The exponential map}

The computation of the exponential map for Stieltjes differential equations with positive derivator is a feat that was achieved in [7]. Here we generalize the construction of the exponential map for the case of a derivator $g$ which is allowed to change sign.

Let $g \in \mathrm{CV}_{-}(I, \mathbb{R})$. In this section, we study the initial value problem for the linear $g$ differential equation:

$$
\begin{aligned}
& x_{g}^{\prime}(t)=c(t) x(t) \quad\left|\mu_{g}\right| \text {-a.e. } t \in I, \\
& x(a)=1 .
\end{aligned}
$$

To this aim, we introduce an exponential map of $c$ associated to $g$.

Definition 7.1 Let $c \in \mathrm{L}_{g}^{1}([a, b))$ be such that

$$
\begin{gathered}
1+c(t)\left[g\left(t^{+}\right)-g(t)\right]>0, \quad t \in[a, b) \cap D_{g} \quad \text { and } \\
\sum_{t \in[a, b) \cap D_{g}}\left|\ln \left(1+c(t)\left[g\left(t^{+}\right)-g(t)\right]\right)\right|<\infty .
\end{gathered}
$$


We define the g-exponential of $c$ on $I, e_{g, c, a}: I \rightarrow \mathbb{R}^{+}$, by

$$
e_{g, c, a}(t):=e^{\int_{[a, t)} \widetilde{c}(s) \mathrm{d} \mu_{g}},
$$

where

$$
\widetilde{c}(t):= \begin{cases}c(t) & \text { if } t \in I \backslash D_{g}, \\ \frac{\ln \left(1+c(t)\left(g\left(t^{+}\right)-g(t)\right)\right)}{g\left(t^{+}\right)-g(t)} & \text { if } t \in I \cap D_{g} .\end{cases}
$$

The proof of the following lemma is essentially the same as in [7, Lemma 6.2], changing $g\left(s^{+}\right)-g(s)$ by $\left|g\left(s^{+}\right)-g(s)\right|$ since $g$ is nonmonotonic now.

Lemma 7.2 The map $\tilde{c}$ in Definition 7.1 is such that $\widetilde{c} \in \mathrm{L}_{g}^{1}([a, b))$. So $e_{g, c, a}$ is well defined.

Proof

$$
\begin{aligned}
\int_{[a, b)}|\widetilde{c}(s)| \mathrm{d}\left|\mu_{g}\right| & =\int_{[a, b) \backslash D_{g}}|\widetilde{c}(s)| \mathrm{d} \mu_{g}+\int_{[a, b) \cap D_{g}}|\widetilde{c}(s)| \mathrm{d}\left|\mu_{g}\right| \\
& =\int_{[a, b) \backslash D_{g}}|c(s)| \mathrm{d}\left|\mu_{g}\right|+\sum_{s \in[a, b) \cap D_{g}}|\widetilde{c}(s)|\left|g\left(s^{+}\right)-g(s)\right| \\
& =\int_{[a, b) \backslash D_{g}}|c(s)| \mathrm{d}\left|\mu_{g}\right|+\sum_{s \in[a, b) \cap D_{g}}\left|\ln \left(1+c(s)\left[g\left(s^{+}\right)-g(s)\right]\right)\right|<\infty,
\end{aligned}
$$

because $c \in \mathrm{L}_{g}^{1}([a, b))$ and $\sum_{t \in[a, b) \cap D_{g}}\left|\ln \left(1+c(t)\left[g\left(t^{+}\right)-g(t)\right]\right)\right|<\infty$.

The $g$-exponential gives us a solution of (7.1).

Theorem 7.3 Let $c \in \mathrm{L}_{g}^{1}([a, b))$. Then, $e_{g, c, a}$ solves the initial value problem (7.1). Furthermore,

$$
e_{g, c, a}(t)=1+\int_{[a, t)} c(s) e_{g, c, a}(s) \mathrm{d} \mu_{g} \quad \text { for every } t \in I .
$$

Proof By Lemma 7.2, $\widetilde{c} \in \mathrm{L}_{g}^{1}([a, b))$. Therefore, by Theorem 6.3, for $h(t)=\int_{[a, t)} \widetilde{c}(s) \mathrm{d} \mu_{g}$, one has $h_{g}^{\prime}=\tilde{c}\left|\mu_{g}\right|$-a.e. on $I$. Hence, by Theorem 6.7, $h \in \mathrm{AC}_{g}(I, \mathbb{R})$. Lemma 6.8 implies that we have that $e_{g, c, a} \in \operatorname{AC}_{g}(I, \mathbb{R})$. Furthermore, by Corollary 5.5, we can apply the chain rule and we get

$$
\left(e_{g, c, a}\right)_{g}^{\prime}(t)=e_{g, c, a}(t) h_{g}^{\prime}(t)=e_{g, c, a}(t) \widetilde{c}(t)=e_{g, c, a}(t) c(t) \quad\left|\mu_{g}\right| \text {-a.e. on } I \backslash D_{g} .
$$

Also, for $t \in D_{g}$, it is easy to verify that

$$
\left(e_{g, c, a}\right)_{g}^{\prime}(t)=\frac{e_{g, c, a}\left(t^{+}\right)-e_{g, c, a}(t)}{g\left(t^{+}\right)-g(t)}=e_{g, c, a}(t) c(t)
$$

Now,

$$
e_{g, c, a}(a)=e^{\int_{[a, a)} \widetilde{c}(s) \mathrm{d} \mu_{g}}=1,
$$


and, since $e_{g, c, a} \in \operatorname{AC}_{g}(I, \mathbb{R})$, by Theorem 6.7,

$$
e_{g, c, a}(t)=1+\int_{[a, t)} c(s) e_{g, c, a}(s) \mathrm{d} \mu_{g} \quad \text { for every } t \in I .
$$

Now that we have covered the case (7.2), we can move to a more general one. In order to do that, consider $T_{c}^{-}:=\left\{t \in[a, b) \cap D_{g}: 1+c(t)\left[g\left(t^{+}\right)-g(t)\right]<0\right\}$. Since $c \in \mathrm{L}_{g}^{1}([a, b))$, it can be shown that the set $T_{c}^{-}$is finite (see [7, Lemma 6.4]), so assume $T_{c}^{-}=\left\{t_{1}, \ldots, t_{m}\right\}$.

Definition 7.4 Let $c \in \mathrm{L}_{g}^{1}([a, b))$ be such that

$$
\begin{gathered}
1+c(t)\left[g\left(t^{+}\right)-g(t)\right] \neq 0, \quad t \in[a, b) \cap D_{g} \quad \text { and } \\
\sum_{t \in[a, b) \cap D_{g}}|\ln | 1+c(t)\left[g\left(t^{+}\right)-g(t)\right] \|<\infty .
\end{gathered}
$$

We define the g-exponential of $c$ on $I, e_{g, c, a}: I \rightarrow \mathbb{R}^{+}$, as

$$
e_{g, c, a}(t):= \begin{cases}e^{\int_{[a, t)} \widetilde{c}(s) \mathrm{d} \mu_{g}} & \text { if } t \in\left[a, t_{1}\right], \\ (-1)^{k} e^{\int_{[a, t)} \widetilde{\tau}(s) \mathrm{d} \mu_{g}} & \text { if } t \in\left(t_{k}, t_{k+1}\right], k=1, \ldots, m,\end{cases}
$$

where

$$
\widetilde{c}(t):= \begin{cases}c(t) & \text { if } t \in I \backslash D_{g}, \\ \frac{\ln \left|1+c(t)\left(g\left(t^{+}\right)-g(t)\right)\right|}{g\left(t^{+}\right)-g(t)} & \text { if } t \in I \cap D_{g} .\end{cases}
$$

Remark 7.5 We use the same notation as in Definition 7.1, particularly $e_{g, c, a}$ and $\widetilde{c}$, because the functions in Definitions 7.1 and 7.4 coincide when (7.2) holds.

Observe that Theorem 7.3 also holds when condition (7.3) holds as a direct consequence of Theorem 7.3 holding for condition (7.2) and the fact of $T_{c}^{-}$being finite.

Finally, we can take this generalization one step further.

Definition 7.6 Let $c \in \mathrm{L}_{g}^{1}([a, b))$ be such that

$$
\sum_{t \in\left[a, t_{0}\right) \cap D_{g}}|\ln | 1+c(t)\left[g\left(t^{+}\right)-g(t)\right]||<\infty
$$

where $t_{0}=a$ if $T_{c}^{0}:=\left\{t \in[a, b) \cap D_{g}: 1+c(t)\left[g\left(t^{+}\right)-g(t)\right]=0\right\}=\emptyset$ and $t_{0}=\inf T_{c}^{0}$ otherwise. We define the g-exponential of $c$ on $I, e_{g, c, a}: I \rightarrow \mathbb{R}^{+}$, by

$$
e_{g, c, a}(t):= \begin{cases}e^{\int_{[a, t)} \widetilde{c}(s) \mathrm{d} \mu_{g}}, & t \in\left[a, t_{1}\right] \cap\left[a, t_{0}\right], \\ (-1)^{k} e^{\int[a, t)} \tilde{c}(s) \mathrm{d} \mu_{g}, & t \in\left(t_{k}, t_{k+1}\right] \cap\left[a, t_{0}\right], k=1, \ldots, m, \\ 0, & t \in\left(t_{0}, b\right],\end{cases}
$$

where $\tilde{c}$ is defined as in (7.4).

Observe that Theorem 7.3 also holds when condition (7.5) holds. The set $T_{c}^{0}$ is also finite, so we could write $t_{0}=\min T_{c}^{0}$. 


\section{Existence results}

In this section, we present an existence result for the following system of $g$-differential equations:

$$
\begin{aligned}
& x_{g}^{\prime}(t)=f(t, x(t)) \quad \mu_{g} \text {-a.e. } t \in\left[t_{0}, t_{0}+\tau\right], \\
& x(0)=x_{0},
\end{aligned}
$$

where $I=\left[t_{0}, t_{0}+T\right]$ and $g=\left(g_{1}, \ldots, g_{n}\right): I \rightarrow \mathbb{R}^{n}$ is such that $g_{j} \in \mathrm{CV}_{-}(I, \mathbb{R})$ for every $j=$ $1, \ldots, n$.

Similarly to the definition given in [7], a notion of $g$-Carathéodory function can be introduced in our context.

Definition 8.1 Let $X$ be a nonempty subset of $\mathbb{R}^{n}$. We say that $f=\left(f_{1}, \ldots, f_{m}\right): I \times X \rightarrow \mathbb{R}^{m}$ is $g$-Carathéodory if it satisfies the following conditions for every $i=1, \ldots, m$ :

(i) for every $x \in X, f_{i}(\cdot, x)$ is $\mu_{g_{i}}$-measurable;

(ii) for $\mu_{g_{i}}$-a.e. $t \in I, f_{i}(t, \cdot)$ is continuous on $X$;

(iii) for every $r>0$, there exists $h_{i, r} \in \mathrm{L}_{g_{i}}^{1}\left(\left[t_{0}, t_{0}+T\right)\right.$, $\left.[0, \infty)\right)$ such that

$$
\left|f_{i}(t, x)\right| \leq h_{i, r}(t) \quad \text { for } \mu_{g_{i}} \text {-a.e. } t \in\left[t_{0}, t_{0}+T\right) \text {, for all } x \in X \text { with }\|x\| \leq r \text {. }
$$

We define $\mathrm{BC}_{g}\left(I, \mathbb{R}^{n}\right):=\prod_{k=1}^{n} \mathrm{BC}_{g_{k}}(I, \mathbb{R})$ with the supremum norm (let us recall that $\mathrm{BC}_{g_{k}}(I, \mathbb{R})=\mathrm{BC}\left(\left(I, \tau_{g_{k}}\right), \mathbb{R}\right)$, where $\tau_{g_{k}}$ is the topology generated by $\left.g_{k}\right)$. Arguing as in [7, Lemma 7.2], it can be shown that the composition $f(\cdot, x(\cdot))$ is in $\mathrm{L}_{g}^{1}\left(\left[t_{0}, t_{0}+T\right), \mathbb{R}^{m}\right):=$ $\prod_{i=k}^{m} \mathrm{~L}_{g_{k}}^{1}\left(\left[t_{0}, t_{0}+T\right), \mathbb{R}\right)$ for every $x \in \mathrm{BC}_{g}\left(I, \mathbb{R}^{n}\right)$.

Lemma 8.2 Let $X$ be a nonempty subset of $\mathbb{R}^{n}$ and $f: I \times X \rightarrow \mathbb{R}^{m}$ a g-Carathéodory function. Then, for every $x \in \mathrm{BC}_{g}\left(I, \mathbb{R}^{n}\right)$, the map $f(\cdot, x(\cdot))$ is in $\mathrm{L}_{g}^{1}\left(\left[t_{0}, t_{0}+T\right), \mathbb{R}^{m}\right)$.

Now, we can establish the existence of $x=\left(x_{1}, \ldots, x_{n}\right)$ a $g$-absolutely continuous solution of (8.1), i.e., $x_{i}$ is $g_{i}$-absolutely continuous for every $i=1, \ldots, n$. The proof of the next theorem is analogous to that of [13, Theorem 4.5]. We present it here in detail for sake of completeness.

Theorem 8.3 Let $r \in \mathbb{R}^{+}$and $f:\left[t_{0}, t_{0}+T\right] \times \overline{B\left(x_{0}, r\right)} \rightarrow \mathbb{R}^{n}$ a g-Carathéodory function. Then there exists $\tau \in(0, T]$ such that (8.1) has a g-absolutely continuous solution defined on $\left[t_{0}, t_{0}+\tau\right]$.

Proof Let $R:=r+\left\|x_{0}\right\|$. Since $f$ is $g$-Carathéodory, we have that, for every $j=1, \ldots, n$, there exists a function $h_{j} \in \mathrm{L}_{g_{j}}^{1}\left(\left[t_{0}, t_{0}+T\right),[0, \infty)\right)$ such that $\left|f_{j}(t, x)\right| \leq h_{j}(t)$, for $\mu_{g_{j}}$-a.e. $t \in\left[t_{0}, t_{0}+T\right)$ and all $x \in X$ with $\|x\| \leq r$. Fix $\tau \in(0, T]$ such that

$$
\max _{j=1, \ldots, n} \int_{\left[t_{0}, t_{0}+\tau\right)} h_{j} \mathrm{~d}\left|\mu_{g_{j}}\right| \leq r
$$

Let

$$
X:=\left\{u \in \mathrm{BC}_{g}\left(\left[t_{0}, t_{0}+\tau\right), \mathbb{R}^{n}\right):\left\|u-x_{0}\right\|_{\infty} \leq r\right\}
$$


The set $X$ is a nonempty closed convex subset of $\mathrm{BC}_{g}\left(\left[t_{0}, t_{0}+\tau\right), \mathbb{R}^{n}\right)$. For $u \in \mathrm{BC}_{g}\left(\left[t_{0}, t_{0}+\right.\right.$ $\left.\tau), \mathbb{R}^{n}\right)$ define $F(u)=\left(F_{1}(u), \ldots, F_{n}(u)\right)$ with

$$
F_{j}(u)=x_{j, 0}+\int_{\left[t_{0}, t_{0}+t\right)} f_{j}(s, u(s)) \mathrm{d} \mu_{g_{j}}, \quad \text { for } j=1, \ldots, n .
$$

The previous lemma implies that $F$ is well defined. It is easy to verify that every fixed point of $F$ is a solution of (8.1) and vice-versa.

Now, we prove certain properties of $F$ that will allow us to use Schauder's Fixed Point Theorem.

- F maps $X$ to $X$.

Let us show that $F u \in \mathrm{BC}_{g}\left(\left[t_{0}, t_{0}+\tau\right), \mathbb{R}^{n}\right)$ for $u \in \mathrm{BC}_{g}\left(\left[t_{0}, t_{0}+\tau\right), \mathbb{R}^{n}\right)$. Let $u \in \mathrm{BC}_{g}\left(\left[t_{0}, t_{0}+\right.\right.$ $\left.\tau), \mathbb{R}^{n}\right)$. By Lemma 8.2, we have that $f(\cdot, u(\cdot)) \in \mathrm{L}_{g}^{1}\left(\left[t_{0}, t_{0}+\tau\right), \mathbb{R}^{n}\right)$. Thus, by Theorem 6.2, we have that $F u \in \mathrm{BC}_{g}\left(\left[t_{0}, t_{0}+\tau\right), \mathbb{R}^{n}\right)$.

Let us show that $F u \in X$ for $u \in X$. Let $u \in X$. We have that $\left\|u-x_{0}\right\| \leq r$. Then, for any $j=1, \ldots, n$ and $t \in\left[t_{0}, t_{0}+\tau\right)$,

$$
\left|\left(F u-x_{0}\right)_{j}(t)\right| \leq \int_{\left[t_{0}, t\right)}\left|f_{j}(s, u(s))\right| \mathrm{d}\left|\mu_{g_{j}}\right|(s) \leq \int_{\left[t_{0}, t_{0}+\tau\right)} h_{j} \mathrm{~d}\left|\mu_{g_{j}}\right| \leq r
$$

so $F u \in X$.

- $F$ is continuous.

Let $\left(u_{n}\right)_{n \in \mathbb{N}} \subset X, u \in X$ be such that $u_{n} \rightarrow u$. Then $u_{n}(s) \rightarrow u_{n}(s)$ for every $s \in\left[t_{0}, t_{0}+\tau\right)$, and, since $f_{j}$ is $g_{j}$-Carathéodory, $f_{j}\left(s, u_{n}(s)\right) \rightarrow f_{j}(s, u(s)) \mu_{g_{j}}$-a.e. Now, for any $j=1, \ldots, n$ and $t \in\left[t_{0}, t_{0}+\tau\right)$,

$$
\left|F_{j} u_{n}(t)-F_{j} u(t)\right| \leq \int_{\left[t_{0}, t_{0}+\tau\right)}\left|f_{j}\left(s, u_{n}(s)\right)-f_{j}(s, u(s))\right| \mathrm{d}\left|\mu_{g_{j}}\right|(s) \leq 2 r
$$

Thus, by the Lebesgue Dominated Convergence Theorem,

$$
\begin{aligned}
\lim _{n \rightarrow \infty}\left\|F u_{n}-F u\right\|_{\infty} & \leq \lim _{n \rightarrow \infty} \int_{\left[t_{0}, t_{0}+\tau\right)}\left|f_{j}\left(s, u_{n}(s)\right)-f_{j}(s, u(s))\right| \mathrm{d}\left|\mu_{g_{j}}\right| \\
& \leq \int_{\left[t_{0}, t_{0}+\tau\right)} \lim _{n \rightarrow \infty}\left|f_{j}\left(s, u_{n}(s)\right)-f_{j}(s, u(s))\right| \mathrm{d}\left|\mu_{g_{j}}\right|=0
\end{aligned}
$$

because $f_{j}\left(s, u_{n}(s)\right) \rightarrow f_{j}(s, u(s)) \mu_{g_{j}}$-a.e.

- $F$ is compact.

Since $X$ is bounded $F(X) \subset X$ is uniformly bounded. Furthermore, $F(X)$ is uniformly $g$-equicontinuous. Indeed, let $\varepsilon \in \mathbb{R}^{+}$and $j=1, \ldots, n$. Since $h_{j} \in \mathrm{L}_{g_{j}}^{1}\left(\left[t_{0}, t_{0}+\tau\right), \mathbb{R}\right)$, we have that there exists $\delta \in \mathbb{R}^{+}$such that if $\left|\mu_{g_{j}}\right|(E)<\delta$, then $\int_{E} f_{j}(s, u(s)) \mathrm{d}\left|\mu_{g_{j}}\right|(s)<\varepsilon$. Hence, if $t, s \in\left[t_{0}, t_{0}+\tau\right)$ are such that $\operatorname{var}_{g_{j}}[t, s]=\left|\mu_{g_{j}}\right|([t, s])<\delta$, then

$$
\left|F_{j} u(t)-F_{j} u(s)\right|=\left|\int_{[s, t)} f_{j}(s, u(s)) \mathrm{d} \mu_{g_{j}}(s)\right| \leq \int_{[s, t)} h_{j} \mathrm{~d}\left|\mu_{g_{j}}\right|<\varepsilon
$$

So, $F(X)$ is uniformly $g$-equicontinuous. It follows from Corollary 4.15 that $F(X)$ is precompact and hence, $F$ is a compact operator. 
Hence, we are in the hypotheses of Schauder's Fixed Point Theorem and we can ensure that $F$ has a fixed point.

Other results similar to Theorem 8.3 can be proven in an standard way. The reader may refer, for instance, to Theorems 4.2, 4.3, 4.4, and 4.8 in [13].

\section{An application to fluid stratification on buoyant miscible jets and plumes}

In [2], the authors modeled fluid stratification on buoyant miscible jets and plumes using the classical model of Morton et al. [18], which is given by

$$
\begin{aligned}
& \left(b^{2} w\right)^{\prime}=2 \alpha b w, \\
& \left(b^{2} w^{2}\right)^{\prime}=2 g \lambda^{2} b^{2} \theta, \\
& \left(b^{2} w \theta\right)^{\prime}=\rho^{\prime} \frac{b^{2} w}{\Lambda \rho_{b}},
\end{aligned}
$$

where the independent variable, $z$, is the height, $b(z)$ the jet width, $w(z)$ the vertical jet velocity, $\theta(z)=\left(\rho(z)-\rho_{j}(z)\right) / \rho_{b}$ the density anomaly, $\rho(z)$ the ambient background density, $g$ the acceleration due to gravity, $\alpha$ the entrainment coefficient, $\lambda$ the mixing coefficient, and $\Lambda=\lambda^{2}\left(1+\lambda^{2}\right)$. With the change of variables $q=b^{2} w, m=b^{4} w^{4}$, and $\beta=b^{2} w \theta$ (volume, momentum, and buoyancy fluxes), (9.1) becomes

$$
\begin{aligned}
& q^{\prime}=2 \alpha m^{\frac{1}{4}}, \\
& m^{\prime}=4 g \lambda^{2} q \beta, \\
& \beta^{\prime}=\rho^{\prime} \frac{q}{\Lambda \rho_{b}} .
\end{aligned}
$$

In [2], the authors consider the particular case where $\rho$ suffers a sudden change at $z=L$ (a jump) and thus $\rho^{\prime}$ can be approximated by a Dirac delta function at $L$ (see [2, Eq. (2.35c)]). In our approach, we will not consider distributions in order to obtain jumps. We will just consider the $\rho$ derivative of $\beta$ which will codify any possible behavior of $\rho$. Hence, this will lead us to consider the system of Stieltjes differential equations

$$
\begin{aligned}
& q^{\prime}=f_{1}(q, m, \beta)=A m^{\frac{1}{4}}, \\
& m^{\prime}=f_{2}(q, m, \beta)=B q \beta, \\
& \beta_{\rho}^{\prime}=f_{3}(q, m, \beta)=C q,
\end{aligned}
$$

where $A=2 \alpha, B=4 g \lambda^{2}$ and $C=1 /\left(\Lambda \rho_{b}\right)$. This is a system of differential equations with $g_{j}$-derivatives where $g_{1}$ and $g_{2}$ are the identity and $g_{3}=\rho \in \mathrm{CV}_{-}\left(\mathbb{R}, \mathbb{R}^{+}\right)$. Now fix $x_{0}=$ $\left(q_{0}, m_{0}, \beta_{0}\right) \in\left(\mathbb{R}^{+}\right)^{3}$. It is clear that there exists $r \in \mathbb{R}^{+}$such that $f_{j}:[0, T] \times \overline{B\left(x_{0}, r\right)} \rightarrow \mathbb{R}$ is $g_{j}$-Carathéodory (just take $\left.r<m_{0}\right)$. Thus, by Theorem 8.3, there exists $\tau \in(0, T]$ such that (9.3) has a $g$-absolutely continuous solution defined on $\left[t_{0}, t_{0}+\tau\right]$. 


\section{Funding}

Marlène Frigon was partially supported by NSERC Canada. F. Adrián F. Tojo was partially supported by Ministerio de Economía y Competitividad, Spain, and FEDER, project MTM2013-43014-P, and by the Agencia Estatal de Investigación (AEI) of Spain under grant MTM2016-75140-P, co-financed by the European Community fund FEDER.

\section{Availability of data and materials}

Not applicable.

\section{Competing interests}

The authors declare that they have no competing interests.

\section{Authors' contributions}

All authors contributed equally to the different parts of the manuscript. All authors read and approved the final manuscript.

\section{Author details}

'Département de mathématiques et de statistique, Université de Montréal, Montréal, Canada. ${ }^{2}$ Instituto de Matemáticas, Facultade de Matemáticas, Universidade de Santiago de Compostela, Santiago de Compostela, Spain.

\section{Publisher's Note}

Springer Nature remains neutral with regard to jurisdictional claims in published maps and institutional affiliations.

\section{Received: 12 November 2019 Accepted: 19 February 2020 Published online: 28 February 2020}

\section{References}

1. Benedetto, J.J., Czaja, W.: Integration and Modern Analysis. Birkhäuser Boston, Boston (2009)

2. Camassa, R., Lin, Z., McLaughlin, R.M., Mertens, K., Tzou, C., Walsh, J., White, B.: Optimal mixing of buoyant jets and plumes in stratified fluids: theory and experiments. J. Fluid Mech. 790, 71-103 (2016)

3. Corduneanu, C.: Integral Equations and Stability of Feedback Systems. Academic Press, New York (1973)

4. Daniell, P.J.: Differentiation with respect to a function of limited variation. Trans. Am. Math. Soc. 19, 353-362 (1918)

5. De Amo, E., Carrillo, M.D., Sánchez, J.F.: Functions with unusual differentiability properties. An. Ştiinţ. Univ. 'Al.I. Cuza' laşi, Mat. 62(1), 121-140 (2016)

6. Dieudonné, J.: Foundations of Modern Analysis, vol. 10. Academic Press, New York (1969)

7. Frigon, M., López Pouso, R.: Theory and applications of first-order systems of Stieltjes differential equations. Adv. Nonlinear Anal. 6(1), 13-36 (2017)

8. Garg, K.M.: Relativization of some aspects of the theory of functions of bounded variation. Diss. Math. 320, 123 (1992)

9. Hart, K.P., Nagata, J.i., Vaughan, J.E.: Encyclopedia of General Topology. Elsevier, Amsterdam (2004)

10. Kreuzer, A.P.: Bounded variation and the strength of Helly's selection theorem. Log. Methods Comput. Sci. 10(4), 1-23 (2014)

11. Kurzweil, J.: Generalized ordinary differential equations and continuous dependence on a parameter. Czechoslov. Math. J. 7(82), 418-449 (1957)

12. López Pouso, R., Márquez Albés, I.: General existence principles for Stieltjes differential equations with applications to mathematical biology. J. Differ. Equ. 264(8), 5388-5407 (2018)

13. López Pouso, R., Márquez Albés, I.: Systems of Stieltjes differential equations with several derivators. Mediterr. J. Math. 1651 (2019)

14. López Pouso, R., Rodríguez, A.: A new unification of continuous, discrete, and impulsive calculus through Stieltjes derivatives. Real Anal. Exch. 40(2), 319-353 (2015)

15. Márquez Albés, I., Tojo, F.: (2019) https://arxiv.org/abs/2001.00467. (arXiv preprint)

16. Monteiro, G.A., Satco, B.: Distributional, differential and integral problems: equivalence and existence results. Electron. J. Qual. Theory Differ. Equ. 2017, 7, 1-26 (2017)

17. Monteiro, G.A., Slavík, A., Tvrdý, M.: Kurzweil-Stieltjes Integral: Theory and Applications, Series in Real Analysis, vol. 15. World Scientific, Hackensack (2019)

18. Morton, B.R., Taylor, G.I., Turner, J.S.: Turbulent gravitational convection from maintained and instantaneous sources Proc. R. Soc. Lond. A 234, 1-23 (1956)

19. Przeradzki, B.: The existence of bounded solutions for differential equations in Hilbert spaces. Ann. Pol. Math. 56(2), 103-121 (1992)

20. Schwabik, Š.: Generalized differential equations. Fundamental results. Rozpravy Československé Akad. Věd / Řada Mat. Přirod. Věd 95(6), 103 (1985)

21. Schwabik, Š., Tvrdý, M., Vejvoda, O.: Differential and Integral Equations. Reidel, Dordrecht (1979)

22. Vala, K.: On compact sets of compact operators. Ann. Acad. Sci. Fenn., Ser. A I(351) (1964) 